
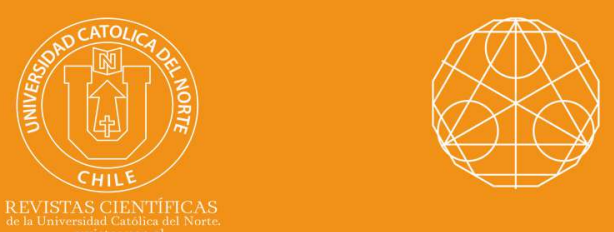

\title{
Applications of proportional calculus and a non-Newtonian logistic growth model
}

\author{
Manuel Pinto ${ }^{1}$ (1) orcid.org/0000-0002-6466-213X \\ Ricardo Torres ${ }^{2}$ (1) orcid.org/0000-0001-6464-0865 \\ William Campillay-Llanos ${ }^{3}$ (1) orcid.org/0000-0002-2853-080X \\ Felipe Guevara-Morales ${ }^{4}$ (10 orcid.org/0000-0002-9473-9548
}

${ }^{1}$ Universidad de Chile, Dept. de Matemáticas, Fc. de Ciencias, Santiago, Chile.

— pintoj@uchile.cl

${ }^{2}$ Universidad Austral de Chile, Inst. de Cs. Físicas y Matemáticas, Fc. de Ciencias, Valdivia, Chile.

घricardo.torres@uach.cl

${ }^{3}$ Universidad Católica del Maule, Dept de Matemática, Físicay Estadística, Fc. deCs. Básicas, Talca, Chile.

williamcampillay@gmail.com

${ }^{4}$ Universidad de Atacama, Dept. de Matemática, Copiapó, Chile.

felipe.guevara@uda.cl

Received: October 2019 | Accepted: August 2020

Abstract:

On the set of positive real numbers, multiplication, represented by $\oplus$, is considered as an operation associated with the notion of sum, and the operation $a \odot b=\operatorname{aln}(b)$ represents the meaning of the traditional multiplication. The triple $(R+, \oplus, \odot)$ forms an ordered and complete field in which derivative and integration operators are defined analogously to the Differential and Integral Calculus. In this article, we present the proportional arithmetic and we construct the theory of ordinary proportional differential equations. A proportional version of Gronwall inequality, Gompertz's function, the $q$-Periodic functions, proportional heat, and wave equations as well as a proportional version of Fourier's series are presented. Furthermore, a non-Newtonian logistic growth model is proposed.

Keywords: Proportional arithmetic; Proportional calculus and proportional derivative and integral ; Geometric difference; Geometric integer; Proportional differential equations; Proportional wave equation; Proportional heat equation; Proportional logistic growth.

MSC (2020): 26A15, 26A24, 26A42, 34A30, 34K13, 34K25, 35A08, 35A09, $42 \mathrm{~A} 16$.

Cite this article as (IEEE citation style):

M. Pinto, R. Torres, W. Campillay-LLanos, and F. Guevara-Morales, "Applications of proportional calculus and a non-Newtonian logistic growth model", Proyecciones (Antofagasta, On line), vol. 39, no. 6, pp. 1471-1513, Dec. 2020, doi: 10.22199/issn.07176279-2020-06-0090.

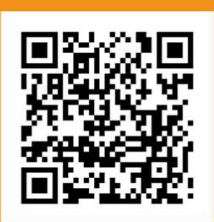

Article copyright: (C) 2020 Manuel Pinto, Ricardo Torres, William Campillay, and Felipe Guevara. This is an open access article distributed under the terms of the Creative Commons License, which permits unrestricted use and distribution provided the original author and source are credited. 


\section{Introduction}

Since the discoveries of Isaac Newton and Gottfried Wilhelm Leibnitz of Differential and Integral Calculus, the representation of natural phenomena by means of differential equations has been a great achievement to understand how nature works. For example, evolution equations and equations of mathematical physics (see [40, 24, 25, 26]). By changing the way these equations are formulated using new arithmetic operations, symmetry of the mathematical equations arises (see [17, 18, 22, 23]). These modifications generate different realizations of mathematical objects (see $[11,12])$. In a broader context, it is considered a bijection $f: X \rightarrow Y \subseteq$ $\mathbf{R}$, where $X$ is a set, $\mathbf{R}$ is the set of real numbers (see $[7,8]$ ). The function $f$ allows us to define an addition, subtraction, multiplication and division in $X$ as follows:

$$
\begin{aligned}
& x \oplus y=f^{-1}(f(x)+f(y)), \\
& x \ominus y=f^{-1}(f(x)-f(y)), \\
& x \odot y=f^{-1}(f(x) \cdot f(y)), \\
& x \oslash y=f^{-1}(f(x) / f(y)) .
\end{aligned}
$$

We note that if $f$ is the identity function, we obtain the traditional arithmetic. It is important to say that calculus with this new arithmetic is not so popular as the calculus of Newton and Leibnitz although it perfectly answers to all conditions expected from the traditional Calculus theory, and it covers only positive functions. Another non-trivial example can be obtained considering $f$ as the Cantor function (see [20]).

In this study, it is considered $f$ as the natural logarithm function, $Y$ the set of the real numbers and $X$ the set of the positive real numbers. Under these considerations the resulting arithmetic is called proportional arithmetic. Grossman and Katz used this way to operate and they presented the Bigeometric Calculus (see [21]). Similarly, in [2] and [3], Bashirov in- troduces the Multiplicative Calculus and its applications. The proportional calculus that is built on proportional arithmetic has been developed by con- tributions of Córdova-Lepe (see [15, 16, 29]). M. Pinto's research proposes mathematical models with this way of operating quantities (see $[13,33,34])$. The article is organized as follows: In section 2 we present the proportional arithmetic and we also highlight its relevant properties. In section 3 we present the differential and integral operators associated with this way of operating, presenting a summary of its properties. In section 4 applications are shown, presenting an introduction to differential equations constructed 
with this arithmetic. Finally, in section 5 shows a model that describes a type of Logistic Growth. The present research effort is an attempt to show how easily can be exposed the traditional calculus in terms of an alternative arithmetic and its usefulness to solve some complex problems.

\section{Proportional Arithmetic}

In (1.1),(1.2),(1.3),(1.4) and division we consider $X=\mathbf{R}^{+}$as the set of positive real numbers, $Y=\mathbf{R}$ the set of real numbers, $f(x)=\ln (x)=$ $\log _{e}(x)$, the natural logarithm function and $f^{-1}(x)=e^{x}$. So, in this case are defined the operations $x \oplus y, x \ominus y, x \odot y$ and $x \oslash y$ as follows:

$$
x \oplus y=x \cdot y, \quad x \ominus y=\frac{x}{y}, \quad x \odot y=x^{\ln (y)}, \quad x \oslash y=x^{\frac{1}{\ln (y)}} .
$$

The number $e$ is the neutral element for $\odot$, where $a \odot e=a$ and $a \oslash e=a$ holds for all $a \in \mathbf{R}^{+}$. The $\odot$-inverse of $a$ is $a^{\{-1\}}:=e^{\frac{1}{\ln (a)}}$, since $a \odot a^{\{-1\}}=$ $a^{\{-1\}} \odot a=\left[e^{\frac{1}{\ln (a)}}\right]^{\ln (a)}=e$. Considering the following equivalence $a \oslash b=e$ if and only if $a=b$, which actually is another way to define when two quantities are equal. Also, it can be defined $x \odot x \odot \cdots \odot x, n$ times, as $x^{\{n\}}$.

Next, we present the following propositions that can be easily proven:

Proposition 1. Let $\mathbf{R}^{+}$the set of positive real numbers. Then

- $\left(\mathbf{R}^{+}, \oplus\right.$.) is a Abelian group with identity 1.

- $\left(\mathbf{R}^{+} \backslash\{1\}, \odot\right)$ is a Abelian group with identity the transcendent number $e$, if the natural logarithm is considered.

- $\odot$ is distributive over $\oplus$.

It is easy to prove the following theorem

Theorem 2. $\left(\mathbf{R}^{+}, \oplus, \odot\right)$ is a field.

In order to present some novelties this type of arithmetic offers, we present the following example 
Example 3. The equation $x \odot x=x^{\{2\}}=4$, has solution $x=2^{\sqrt{2}}$. Considering the logarithm base 2, we can see that

$$
\begin{array}{r}
x^{\{2\}}=4, \\
x \odot x=4, \\
\log _{2}(x \odot x)=\log _{2} 2^{2}, \\
\left(\log _{2}(x)\right)^{2}=2, \\
\log _{2}(x)=\sqrt{2} .
\end{array}
$$

This number, $x=2^{\sqrt{2}}$, is known as the Gelfond-Schneider's constant. It is important to mention that the challenge of proving the nature of this type of numbers was presented in 1900 by David Hilbert at the World Congress of Mathematicians presents a series of problems that includes this challenge (see $[6])$.

Remark 4. In the proportional arithmetic sense, the number $2^{\sqrt{2}}$ is proportionally algebraic, since it satisfies the relationship $p\left(2^{\sqrt{2}}\right)=1$, where $p(x)=\frac{x^{\{2\}}}{4}$.

\subsection{Proportional Algebraics Curves}

In the following, we propose an application of the proportional arithmetic to build and understand a type of algebraic curves. The proportional line can be described algebraically by

$$
y=[m \odot x] \cdot b
$$

where $a$ is the slope or gradient of the line, $b$ is the $y$-intercept of the line, that is when $x=1$.

This expression of proportional nature has been used in various mathemat- ical works, for example it allows to relate body mass $x(\mathrm{~kg})$, to another characteristic, $y$, of the natural history of organisms. The proportional line has been useful to parameterize systems of differential equations that represent interactions between species, obtaining interesting results (see $[19,30,38]$ ).

The notion of inclination of the proportional line is characterized as follows:

Proposition 5. Let two different points of a proportional line $L$ defined by $L_{1}:=\left(x_{1}, y_{1}\right)$ and $L_{2}:=\left(x_{2}, y_{2}\right)$. The slope $m$ is calculated using proportional arithmetic through the following expression 


$$
m=\frac{y_{1}}{y_{2}} \oslash \frac{x_{1}}{x_{2}}
$$

Proof. The following system of equations can be formed:

$$
\left\{\begin{array}{l}
y_{1}=b \cdot x_{1} \odot m=b \cdot x_{1}^{\ln (m)} \\
y_{2}=b \cdot x_{2} \odot m=b \cdot x_{2}^{\ln (m)}
\end{array}\right.
$$

Comparing the quantities $y_{1}$ and $y_{2}$ by the quotient, we have

$$
\frac{y_{1}}{y_{2}}=\frac{b \cdot x_{1}^{\ln (m)}}{b \cdot x_{2}^{\ln (m)}} \Longrightarrow \ln \left(\frac{y_{1}}{y_{2}}\right)=\ln (m) \cdot \ln \left(\frac{x_{1}}{x_{2}}\right),
$$

so, we conclude that

$$
\frac{\ln \left(\frac{y_{1}}{y_{2}}\right)}{\ln \left(\frac{x_{1}}{x_{2}}\right)}=\ln (m) \Longrightarrow \frac{y_{1}}{y_{2}} \oslash \frac{x_{1}}{x_{2}}=m
$$

Now, we give some examples applying the notion of slope presented before:

1. Case 1: For $m \in] 0,1]$ we consider the functions $(a) y=2,5 \cdot x \odot$ $(0,85),(b) y=0,3 \cdot x \odot(0,5)(c) y=1,5 \cdot x \odot(0,2)$, which acquire a decreasing hyperbolic form.

2. Case 2: For $m=1$, we have a constant function. This is reasonable, since the notion of the number 0 in proportional arithmetic is equivalent to 1 , and its graph is paralell to the $x$ axis.

3. Case 3: For $m \in] 1, e]$ the curves present a concave growth. Figures correspond to the equations $(a) y=3 \cdot x \odot(1,1),(b) y=2 \cdot x \odot(1,9)$ and $(c) y=0,2 \cdot x \odot 2,7$.

4. Case 4: For $m=e$ we have the linear function $(a) y=0.1 \cdot x,(b) y=x$ and $(c) y=5 \cdot x$.

5. Case 5: For $m \in] e, \infty]$ the following curves are presented: $(a) y=$ $0.1 \cdot x^{2},(b) y=x^{3}$ and $(c) y=5 \cdot x^{7}$ with $x>0$. 
In Figure 2.1, graphical representations of the proportional lines are presented
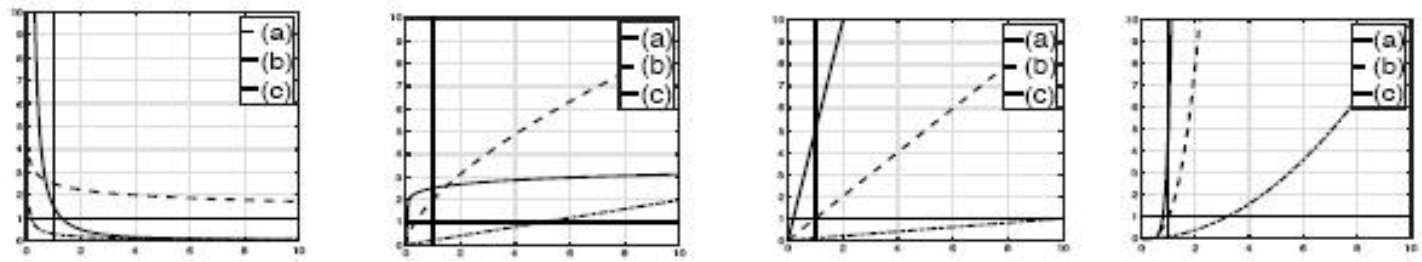

Figure 2.1: Examples of proportional lines. From left to right, cases $1,3,4$ and 5 are presented.

In the proportional cartesian coordinate system (see [11]). The general proportional conic equation has the following form:

$(2.8)\left(A \odot x^{\{2\}}\right) \cdot(B \odot x \odot y) \cdot\left(C \odot y^{\{2\}}\right) \cdot(D \odot x) \cdot(E \odot y) \cdot F=1$,

with $A, B, C, D, E, F$ positives and not all equal to 1 , and let $a, b$ positive real numbers not equal to 1 . In Figure 2, the four types of proportional conics are presented with their respective equations.

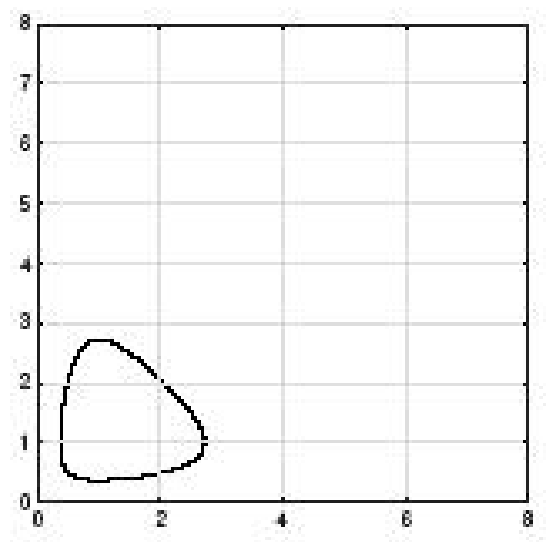

(a) $x^{\{2\}} \cdot y^{\{2\}}=a^{\{2\}}$.

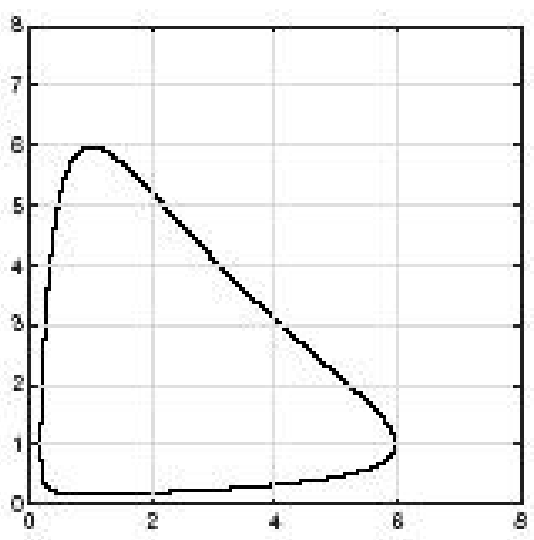

(b) $\left(x^{[2]} \oslash a^{[2]}\right) \cdot\left(y^{[2]} \oslash b^{\{2]}\right)=e$. 


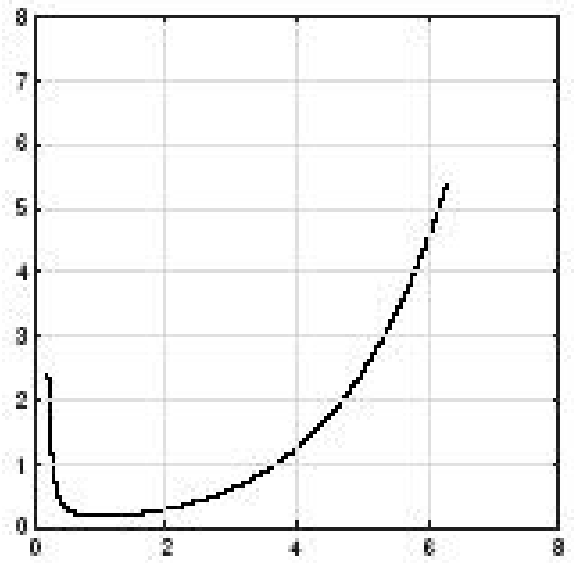

(c) $x^{\{2\}}=e^{4} \odot y \odot a$.

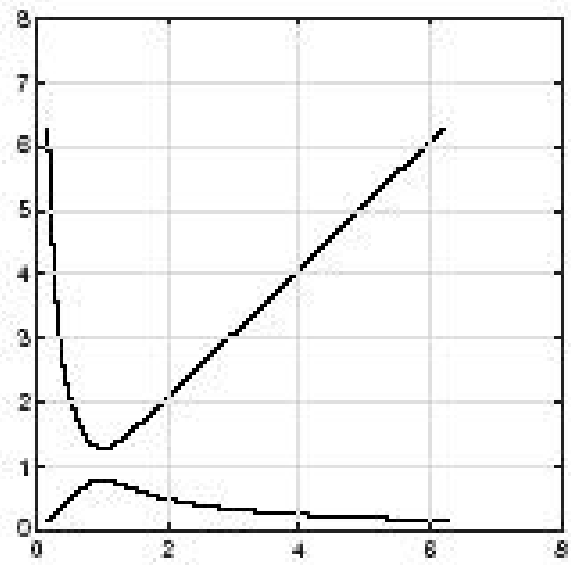

(d) $\frac{x^{\{2\}} \oslash a^{\{2\}}}{y^{\{2\}} \oslash b^{\{2\}}}=e$.

Figure 2.2: Proportional conics. (a) In this case $a=e$. (b) In this case $a=2$ and $b=3.7$. (c) In this case $a=0.1$. (d) In this case $a=3$ and $b=4.5$.

Also, we can give a proportional version of the polynomial $\operatorname{ring} \mathbf{R}^{+}[X]$, in $X$ over a field $\mathbf{R}^{+}$as the set of expressions, called proportional polynomials in $X$ of the form

$$
p(X)=p_{0} \cdot\left(p_{1} \odot X\right) \cdot\left(p_{2} \odot X^{\{2\}}\right) \cdots\left(p_{m-1} \odot X^{\{m-1\}}\right) \cdot\left(p_{m} \odot X^{\{m\}}\right),
$$

where $p_{0}, p_{1}, \ldots, p_{m}$ the coefficients of $p$ are elements of $\mathbf{R}^{+}$and $X, X^{\{2\}}, \ldots, X^{\{m\}}$ are formal symbols (the powers of $X$ ). By convention, $X^{\{0\}}=e, X^{\{1\}}=X$, and the product of the powers of $X$ is defined by the formula $X^{\{k\}} \odot X^{\{l\}}=$ $X^{\{k+l\}}$. According to these definitions it is possible to obtain the following properties:

1. $X \odot Y^{-1}=X^{-1} \odot Y=(X \odot Y)^{-1}$,

2. $Z \odot(X \cdot Y)=(Z \odot X) \cdot(Z \odot Y)$,

3. $\left(\frac{X}{Y}\right)^{\{2\}}=X^{\{2\}} \cdot(X \odot Y)^{-2} \cdot Y^{2}$, 
4. $(X \cdot Y) \odot(X \cdot Z)=X^{\{2\}} \cdot((Y \cdot Z) \odot X) \cdot(Y \odot Z)$,

5. $(X \cdot Y \cdot Z)^{\{2\}}=X^{\{2\}} \cdot Y^{\{2\}} \cdot Z^{\{2\}} \cdot((X \odot Y) \cdot(X \odot Z) \cdot(Y \odot Z))^{2}$,

6. $(X \cdot Y) \odot\left(X \cdot Y^{-1}\right)=(X \odot X) \cdot\left(X \odot Y^{-1}\right) \cdot(Y \odot X) \cdot\left(Y \odot Y^{-1}\right)=$ $X^{\{2\}} \cdot\left(Y^{\{2\}}\right)^{-1}$.

In addition to these properties it is possible to define the proportional binomial and the corresponding binomial coefficients

$$
\begin{gathered}
(X \cdot Y)^{\{1\}}=(X \cdot Y), \\
(X \cdot Y)^{\{2\}}=X^{\{2\}} \cdot(X \odot Y)^{2} \cdot Y^{\{2\}}, \\
(X \cdot Y)^{\{3\}}=X^{\{3\}} \cdot\left(X^{\{2\}} \odot Y\right)^{3} \cdot\left(X \odot Y^{\{2\}}\right)^{3} \cdot Y^{\{3\}}, \\
(X \cdot Y)^{\{4\}}=X^{\{4\}} \cdot\left(X^{\{3\}} \odot Y\right)^{4} \cdot\left(X^{\{2\}} \odot Y^{\{2\}}\right)^{6} \cdot\left(X \odot Y^{\{3\}}\right)^{4} \cdot Y^{\{3\}} .
\end{gathered}
$$

In general, it can be expressed as a product of proportional algebraic expressions $(X \cdot Y)^{\{n\}}=\prod_{k=1}^{n}\left(X^{\{n-k\}} \odot Y^{\{k\}}\right)^{\frac{n !}{)^{! !}(n-k) !}}$ and the well-known triangle of Pascal is appreciated in the exponents.

\subsection{The concept of limit in proportional arithmetic}

The proportional absolute value corresponds to:

$$
[[x]]= \begin{cases}x, & \text { if } x \geq 1 \\ x^{-1}, & \text { if } x \in(0,1) .\end{cases}
$$

If we consider the relative value and the set of positive real numbers, the relative metric is defined as

$$
\begin{array}{r}
d: \mathbf{R}^{+} \times \mathbf{R}^{+} \rightarrow \mathbf{R}^{+}, \\
d(x, y)=\left[\left[\frac{x}{y}\right]\right] .
\end{array}
$$

It is simple to prove that:

- $\left[\left[\frac{x}{y}\right]\right] \geq 1$

- $\left[\left[\frac{x}{y}\right]\right]=1$ if on ly if $x=y$, 
- $\left[\left[\frac{x}{y}\right]\right]=\left[\left[\frac{y}{x}\right]\right]$,

- $\left[\left[\frac{x}{z}\right]\right] \leq\left[\left[\frac{x}{y}\right]\right] \odot\left[\left[\frac{y}{z}\right]\right]$.

In analogy to the geometric limit (see $[5,9])$ we define this notion in terms of proportional arithmetic.

Definition 6. We say that $\lim _{x \rightarrow x_{0}} f(x)=L$, where $L \in \mathbf{R}^{+}$, if only if for all $\epsilon>1$ there exists $\delta>1$ such that $1<\left[\left[\frac{x}{x_{0}}\right]\right]<\delta$, then $\left[\left[\frac{f(x)}{L}\right]\right]<\epsilon$.

According to this definition it is possible to specify the meaning of equality $\lim _{x \rightarrow x_{0}} f(x)=f\left(x_{0}\right)$ and therefore the notion of continuity in the proportional sense.

Definition 7. We say that $f$ is continuous at $x_{0}$ or $\lim _{x \rightarrow x_{0}} f(x)=f\left(x_{0}\right)$, if only if for all $\epsilon>1$ there exists $\delta>1$ such that if $\left[\left[\frac{x}{x_{0}}\right]\right]<\delta$, then $\left[\left[\frac{f(x)}{f\left(x_{0}\right)}\right]\right]<\epsilon$.

In fact, if $f(x)=x^{\{2\}}$, following the definition of the $2^{\text {nd }}$-power of $x$, we have that

$$
\ln (f(x))=(\ln (x))^{2} .
$$

Now, by the continuity of the usual logarithm function, we know that

$$
\lim _{x \rightarrow a} \ln (f(x))=\ln \left(\lim _{x \rightarrow a} f(x)\right) .
$$

Thus, we have

$$
\lim _{x \rightarrow a} \ln (f(x))=\lim _{x \rightarrow a} \ln ^{2}(x)=\ln ^{2}(a)=\ln \left(a^{\ln (a)}\right)=\ln \left(a^{\{2\}}\right) .
$$

Hence

$$
\ln \left(\lim _{x \rightarrow a} f(x)\right)=\ln \left(a^{\{2\}}\right),
$$

so

$$
\lim _{x \rightarrow a} f(x)=a^{\{2\}} .
$$

Last expression is equivalent to

$$
\lim _{x \rightarrow a} \frac{f(x)}{a^{\{2\}}}=1 .
$$

In general $x, x^{\{2\}}, \ldots, x^{\{n\}}$ are continuous and therefore every proportional polynomial is continuous in this sense. 


\section{Proportional Calculus}

\subsection{Proportional Derivative}

The following definitions and theorems can be found in $[15,16]$.

Definition 1 (Proportional Derivatives). If $f$ is a positive function over an interval $I$, we define the following limits:

$$
\tilde{f}\left(x_{0}\right)=\lim _{x \rightarrow x_{0}} \frac{f(x)}{f\left(x_{0}\right)} \oslash \frac{x}{x_{0}}, \quad \text { or } \quad \tilde{f}\left(x_{0}\right)=\lim _{h \rightarrow 1} \frac{f\left(x_{0} \cdot h\right)}{f\left(x_{0}\right)} \oslash h .
$$

If this limit exists, the function $\tilde{f}$ itself is called the proportional derivative of $f$ for which the symbol $\frac{\widetilde{d f}}{d t}$ can also be used. The proportional derivative of $\tilde{f}$ is called second proportional derivative of $f$ and it is denoted by $\widetilde{\widetilde{f}}$. In a similar way the $n^{\text {th }}$ proportional derivative of $f$ can be defined, which is denoted by $\widetilde{f}^{(n)}$ for $n=0,1, \ldots$ with $\widetilde{f}^{(0)}=f$. Note that if $f$ is derivable in $x_{0}$, then it is continuous at $x_{0}$, since it has the following equality

$$
\lim _{x \rightarrow x_{0}} \frac{f(x)}{f\left(x_{0}\right)}=\lim _{x \rightarrow x_{0}}\left[\frac{f(x)}{f\left(x_{0}\right)} \oslash \frac{x}{x_{0}}\right] \odot\left[\frac{x}{x_{0}}\right]
$$

thus $\lim _{x \rightarrow x_{0}} f(x)=f\left(x_{0}\right)$. Next, we present some examples of derivative of functions in the proportional sense

Example 2. The derivative of the function $f(x)=x$, is $\widetilde{f(x)}=e$.

$$
\lim _{x \rightarrow x_{0}} \frac{f(x)}{f\left(x_{0}\right)} \oslash \frac{x}{x_{0}}=\lim _{x \rightarrow x_{0}} \frac{x}{x_{0}} \oslash \frac{x}{x_{0}}=\lim _{x \rightarrow x_{0}} e=e .
$$

Example 3. Let a positive constant. The derivative of the function $f(x)=$ $a \cdot x^{\{2\}}$, is $\widetilde{f(x)}=x^{2}$.

- $\frac{f(x \cdot h)}{f(x)}=\frac{a \cdot\left(x^{\{2\}} \cdot(x \odot h)^{2} \cdot h^{\{2\}}\right)}{\left.a \cdot x^{2}\right\}}=\left(x^{2} \odot h\right) \cdot h^{\{2\}}=h \odot\left(x^{2} \cdot h\right)$,

- $\frac{f(x \cdot h)}{f(x)} \oslash h=\left[h \odot\left(x^{2} \cdot h\right)\right] \oslash h=x^{2} \cdot h$,

$$
\lim _{h \rightarrow 1} \frac{f(x \cdot h)}{f(x)} \oslash h=\lim _{h \rightarrow 1} x^{2} \cdot h=x^{2} .
$$


Example 4. The derivative of the function $f(x)=\cos _{e}(x)=e^{\cos (\ln (x))}$, is $\widetilde{f(x)}=\frac{1}{\sin _{e}(x)}$, where $\sin _{e}(x)=e^{\sin (\ln (x))}$.

$$
\begin{gathered}
\lim _{h \rightarrow 1}\left(\frac{e^{\cos (\ln (x \cdot h))}}{e^{\cos (\ln (x))}}\right)^{\frac{1}{\ln (h)}}=\lim _{h \rightarrow 1}=e^{\frac{\cos (\ln (x)+\ln (h))-\cos (\ln (h))}{\ln (h)}}, \\
=\lim _{h \rightarrow 1} e^{\frac{\cos (\ln (x)) \cdot \cos (\ln (h))-\operatorname{sen}(\ln (x)) \cdot \operatorname{sen}(\ln (h))-\cos (\ln (h))}{\ln (h)}}, \\
=\lim _{h \rightarrow 1} e^{\cos (\ln (x)) \frac{\cos (\ln (h))-1}{\ln (h)}-\operatorname{sen}(\ln (x)) \frac{\operatorname{sen}(\ln (h))}{\ln (h)}} .
\end{gathered}
$$

If we calculate this limit then it is obtained that $\widetilde{\cos _{e}(x)}=e^{-\operatorname{sen}(\ln (x))}=$ $\frac{1}{\operatorname{sen}_{e}(x)}$.

Example 5. The derivative of the function $f(x)=\sin _{e}(x)$, is $\widetilde{f(x)}=$ $\cos _{e}(x)$. It is verified analogously as the previous example.

The definition of the derivative presented before has a conceptual and practical development different from other researches. For example, if the function $f(x)=e^{x}$ is considered, the proportional derivative $f(x)=e^{x}$ is different from the multiplicative derivative $f^{*}(x)=e$ presented in [2]. For the following theorems consider $f, g: \mathbf{R}^{+} \rightarrow \mathbf{R}^{+}$two positive and differentiable functions in the proportional sense:

Theorem 6 (Proportional derivative of a product). The proportional derivative of a product will be given by the formula

$$
\widetilde{f \odot g}=(\widetilde{f} \odot g) \cdot(\widetilde{g} \odot f) .
$$

Example 7. The derivative of the function $f(x)=x^{\{3\}}$, is $\widetilde{f(x)}=\left(x^{\{2\}}\right)^{3}$. To obtain the derivative of this function, it is considered $h(x)=x^{\{2\}}$ and $g(x)=x$ and we use the preceding theorem applied to the functions $h$ and $g$

$\widetilde{h \odot g}=\left(\left(\widetilde{x^{\{2\}}}\right) \odot x\right) \cdot\left(\widetilde{x} \odot x^{\{2\}}\right)=(x \odot x)^{2} \cdot x^{\{2\}}=\left(x^{\{2\}}\right)^{2} \cdot x^{\{2\}}=\left(x^{\{2\}}\right)^{3}$.

In general, the derivative of $x^{\{n\}}$ is $\left(x^{\{n-1\}}\right)^{n}$.

Theorem 8. If $f^{\prime}\left(x_{0}\right)$ is the usual derivative at $x=x_{0}$, then we have:

$$
\widetilde{f}\left(x_{0}\right)=e^{x_{0}\left(\frac{f^{\prime}\left(x_{0}\right)}{f\left(x_{0}\right)}\right)} .
$$


Example 9. The derivative of the function $f(x)=\ln (x)$, is $\widetilde{f(x)}=e \oslash x$.

$$
\widetilde{f}\left(x_{0}\right)=e^{x_{0}\left(\frac{f^{\prime}\left(x_{0}\right)}{f\left(x_{0}\right)}\right)}=e^{\frac{1}{\ln \left(x_{0}\right)}}=e \oslash x_{0} .
$$

Theorem 10. For all $\alpha, \beta \in \mathbf{R}$, we have that:

$$
\widetilde{f^{\alpha} g^{\beta}}\left(x_{0}\right)=\left(\widetilde{f}\left(x_{0}\right)\right)^{\alpha} \cdot\left(\widetilde{g}\left(x_{0}\right)\right)^{\beta} .
$$

Also, the proportional version of the chain rule can be established:

Theorem 11 (The Proportional Chain Rule). Let $f$ be proportional differentiable at $g(x)$ and $g$ proportional differentiable. Then

$$
\widetilde{f \circ g}=(\widetilde{f} \circ g) \odot \widetilde{g} .
$$

Example 12. The derivative of the function $f(x)=e^{x^{2}}$ is $\widetilde{f(x)}=\left(e^{x^{2}}\right)^{2}$. It is clear that the functions considered are $h(x)=e^{x}$ and $g(x)=x^{2}$. Then, applying the proportional chain rule we have

$$
\widetilde{f(x)}=\widetilde{h \circ g}(x)=(\widetilde{h} \circ g)(x) \odot \widetilde{g}(x)=e^{x^{2}} \odot e^{2}=\left(e^{x^{2}}\right)^{2} .
$$

We will give more details of this example see section 4.

Example 13. Let $f$ be a function proportionally derivable then its inverse $f^{-1}$ is proportionally derivable. The derivative of $f^{-1}(x)$ is $e \oslash\left[\widetilde{f}\left(f^{-1}(x)\right)\right]$. This is obtained by considering the equality $f \circ f^{-1}=I$ and using the previous theorem with $g=f^{-1}$.

\subsubsection{Some important proportional theorems related to the deriva- tive}

For the proofs of the following theorems we have combined ideas of [10] and [1]. One of the fundamental theorems related to the derivative is the mean value theorem. Next, we give the proportional version of this very important theorem:

Theorem 14 (Proportional Mean Value Theorem). If $f$ is positive and continuous in $[a, b]$ and proportional differentiable on $(a, b)$, then there exists a number $c$ in $(a, b)$ such as that

$$
\tilde{f}(c)=\frac{f(b)}{f(a)} \oslash \frac{b}{a} .
$$


This theorem is used to prove the following propositions, which will be applied in section 4 .

Proposition 15. If $\widetilde{f(x)}=1$ for all $x \in(a, b)$, then $f(x)=C$ for all $x \in(a, b)$, where $C$ is a constant.

Proposition 16. If $\widetilde{f(x)}=\widetilde{g(x)}$ for all $x \in(a, b)$, then there exists a constant $C$ such that $f(x)=C \cdot g(x)$ for all $x \in(a, b)$.

To conclude this section, we present the study of functions that allows a representation as a Taylor's product. This is, $f$ can be represented as a Taylor's product if it admits the following representation

$f(x)=c_{0} \cdot\left(\left[c_{1} \odot\left(\frac{x}{a}\right)\right] \cdot\left[c_{2} \odot\left(\frac{x}{a}\right)^{\{2\}}\right] \cdot\left[c_{3} \odot\left(\frac{x}{a}\right)^{\{3\}}\right] \ldots\left[c_{n} \odot\left(\frac{x}{a}\right)^{\{n\}}\right]\right)$.

In order to deduce this fact, firstly we will determine the coefficients $c_{n}$. Note that for $f$ we have:

- $\widetilde{f(x)}=c_{1} \cdot\left[c_{2}^{2} \odot\left(\frac{x}{a}\right)\right] \cdot\left[c_{3}^{3} \odot\left(\frac{x}{a}\right)^{\{2\}}\right] \ldots$

- $\widetilde{f}^{(2)}(x)=c_{2}^{2} \cdot\left[c_{3}^{3 \cdot 2} \odot\left(\frac{x}{a}\right)\right] \ldots$

- $\widetilde{f}^{(3)}(x)=c_{3}^{3 \cdot 2 \cdot 1} \ldots$

Evaluating $x=a$ in the preceding equations, we obtain

$$
f(a)=c_{0}, \widetilde{f(a)}=c_{1}^{1 !}, \quad \widetilde{f}^{(2)}(a)=c_{2}^{2 !}, \quad \widetilde{f}^{(3)}(a)=c_{3}^{3 !} .
$$

From this, we deduce that

$$
c_{n}=\left(\widetilde{f}^{(n)}(a)\right)^{\frac{1}{n !}} .
$$

Therefore, we can state the following proposition

Proposition 17. If $f$ is a positive function, with $n^{\text {th }}$ proportional derivatives and representation of Taylor product for all $x$ in a range that contains the positive real number $a$, then the coefficients should be $c_{n}=\left(\widetilde{f}^{(n)}(a)\right)^{\frac{1}{n !}}$. 
Theorem 18 (Proportional Taylor's Theorem). Let $f$ be a function such that $\widetilde{f}^{(n+1)}(x)$ exists for all $x$ in a range that contains the number $a$. Then for all $x$ we have

$$
f(x)=P_{n}(x) \cdot R_{n}(x),
$$

where

$$
\begin{aligned}
P_{n}(x)= & f(a) \cdot\left[(\widetilde{f(a)})^{\frac{1}{1 !}} \odot\left(\frac{x}{a}\right)\right] \cdot\left[\left(\widetilde{f}^{(2)}(a)\right)^{\frac{1}{2 !}} \odot\left(\frac{x}{a}\right)^{\{2\}}\right] \\
& {\left[\left(\widetilde{f}^{(n)}(a)\right)^{\frac{1}{n !}} \odot\left(\frac{x}{a}\right)^{\{n\}}\right], }
\end{aligned}
$$

will be called the proportional Taylor Polynomial of $f$ in $a$, of $n^{\text {th }}$ degree and

$$
R_{n}(x)=\left(\widetilde{f}^{(n+1)}(c)\right)^{\frac{1}{(n+1) !}} \odot\left(\frac{x}{a}\right)^{\{n+1\}}
$$

is called the proportional Lagrange form of the residue. The number $c$ lies between $a$ and $x$.

Theorem 19 (Convergence of a Taylor Series). Suppose that $f$ is a function that has derived from all orders over an interval centered on the number a. If $\lim _{n \rightarrow \infty} R_{n}(x)=1$ for all $x$ in the interval, then the Taylor product generated by $f$ it is convergent and converges to $f(x)$,

$$
f(x)=\prod_{k=0}^{\infty}\left(\widetilde{f}^{(k)}(a)\right)^{\frac{1}{k !}} \odot\left(\frac{x}{a}\right)^{\{k\}} .
$$

Example 20. Consider $f(x)=e^{x}$, and $a=1$. We have, $\widetilde{f}^{(n)}(x)=e^{x}$ and it holds for all $n$, so we have $\widetilde{f}^{(n)}(1)=e$ and the Taylor's product for $f$ is

$$
e^{x}=e \cdot[e \odot x] \cdot\left[e^{\frac{1}{2 !}} \odot x^{\{2\}}\right] \cdot\left[e^{\frac{1}{3 !}} \odot x^{\{3\}}\right] \cdots=\prod_{k=0}^{\infty} e^{\frac{1}{k !} \odot x^{\{k\}}}
$$

This is a convergent product and this convergence is verified by applying natural logarithm to equality and considering the convergent series $\sum_{k=0}^{\infty} \frac{(\ln (x))^{k}}{k !}$. 
Example 21. Consider $f(x)=\cos _{e}(x)$, and $a=1$.

- As $f(x)=\cos _{e}(x)$, then $f(1)=e$,

- As $\widetilde{f(x)}=\frac{1}{\sin _{e}(x)}$, then $\widetilde{f(1)}=1$,

- As $\widetilde{f}^{(2)}(x)=\frac{1}{\cos _{e}(x)}$, then $\widetilde{f}^{(2)}(1)=\frac{1}{e}$,

- As $\tilde{f}^{(3)}(x)=\sin _{e}(x)$, then $\tilde{f}^{(3)}(1)=1$.

Hence, the Taylor's product for $f$ is

$$
\begin{aligned}
\cos _{e}(x) & =e \cdot\left[\left(\frac{1}{e}\right)^{\frac{1}{2 !}} \odot\left(\frac{x}{a}\right)^{\{2\}}\right] \cdot\left[(e)^{\frac{1}{4 !}} \odot\left(\frac{x}{a}\right)^{\{4\}}\right] \cdot\left[\left(\frac{1}{e}\right)^{\frac{1}{6 !}} \odot\left(\frac{x}{a}\right)^{\{6\}}\right] \ldots \\
& =\prod_{k=0}^{\infty}\left(e^{(-1)^{k}}\right)^{\frac{1}{2 k !}} \odot x^{\{2 k\}} .
\end{aligned}
$$

This is a convergent product and this convergence is verified by applying natural logarithm to equality and considering the convergent series $\sum_{k=0}^{\infty} \frac{(-1)^{k}}{2 k !}(\ln (x))^{2 k}$.

\subsection{Proportional Integrals}

The proportional integral of a positive function is a type of product integration (see $[36,37])$. In this section the notion of a integral is constructed analogously as it is constructed in the terms of Darboux's ideas (see [28]). For this, it can be defined an order relation in the set $P$ $([a, b])$ of all par- titions of $[a, b]$, in the same way as usual Riemann integration. In [10], definitions, basic results and theorems have been introduced and are listed below.

Definition 22. Suppose $f$ is positive and bounded on $[a, b]$ and $\Delta=$ $\left\{t_{0}, \ldots, t_{n}\right\}$ is a partition of $[a, b]$. Given $i=1,2, \ldots, n$; define

$$
m_{i}=\inf \left\{f(x): t_{i-1} \leq x \leq t_{i}\right\}, \quad M_{i}=\sup \left\{f(x): t_{i-1} \leq x \leq t_{i}\right\} .
$$

The lower product of $f$ for $\Delta$, is designated $P_{\Delta}^{-}$and it is define as

$$
P_{\Delta}^{-} f=\prod_{i=1}^{n} m_{i} \odot\left(\frac{t_{i+1}}{t_{i}}\right) .
$$


In the same way, the upper product of $f$ for $\Delta$, is designated $P_{\Delta}^{+}$and it is defined as

$$
P_{\Delta}^{+} f=\prod_{i=1}^{n} M_{i} \odot\left(\frac{t_{i+1}}{t_{i}}\right)
$$

Definition 23. A positive and bounded function $f$ on $[a, b]$ is proportionally integrable on $[a, b]$ if

$$
\sup \left\{P_{\Delta}^{-} f\right\}=\inf \left\{P_{\Delta}^{+} f\right\} .
$$

In this case, this number is called the proportional integral of $f$ on $[a, b]$ and it is denoted by

$$
\widetilde{\int_{a}^{b}} f \rho_{x}
$$

It is also possible to give the proportional version of Riemann's criterion for integrability. If $f$ is positive bounded on $[a, b]$, then $f$ is proportional integrable on $[a, b]$ if and only if for all $\varepsilon>1$ exists a partition $\Delta$ of $[a, b]$ such that

$$
\left[\left[\frac{P_{\Delta}^{+} f}{P_{\Delta}^{-} f}\right]\right]<\varepsilon,
$$

for all partition $P$ such that $P>\Delta$.

Example 24. There are functions that are not integrable in the proportional sense.

$$
f(x)= \begin{cases}e & \text { if } x \in \mathbf{Q} \\ 1 & \text { if } x \in \mathbf{Q}\end{cases}
$$

Let $\Delta=\left\{t_{1}, \ldots, t_{n}\right\}$ be any partition of the interval $[a, b]$. Since we know that there is an irrational number between $\left[t_{i}, t_{i+1}\right]$ then $m_{i}=1$ and we also know that there is a rational number between $\left[t_{i-1}, t_{i}\right]$ then $M_{i}=e$. We have

$$
\sup \left\{P_{\Delta}^{-} f\right\}=\inf \left\{P_{\Delta}^{+} f\right\}
$$

because

$$
P_{\Delta}^{-} f=\prod_{i=1}^{n} 1 \odot\left(\frac{t_{i+1}}{t_{i}}\right)=\prod_{i=1}^{n} 1=1
$$

and

$$
P_{\Delta}^{+} f=\prod_{i=1}^{n} e \odot\left(\frac{t_{i+1}}{t_{i}}\right)=\prod_{i=1}^{n}\left(\frac{t_{i+1}}{t_{i}}\right)=\frac{t_{n}}{t_{1}}=\frac{b}{a} .
$$


The following theorems are related with the proportional integrable character of $f$. They are the analogous of the traditional results of the Integral Calculus:

Theorem 25. If $f$ is positive and continuous $[a, b]$, then $f$ is proportional integrable in $[a, b]$.

Theorem 26. Consider $a<b<c$. If $f$ is positive and integrable over $[a, b]$, then $f$ is integrable on $[a, c]$ and $[c, d]$. Conversely, if $f$ is integrable on $[a, c]$ and $[c, b]$, then $f$ is integrable on $[a, b]$. Finally, if $f$ is integrable on $[a, b]$, then

$$
\widetilde{\int_{a}^{b}} f \rho_{x}=\widetilde{\int_{a}^{c}} f \rho_{x} \cdot \widetilde{\int_{c}^{b}} f \rho_{x}
$$

Theorem 27 (Proportional Integration of a Product). If $f$ y $g$ are positive and integrable functions on $[a, b]$, then $f \cdot g$ is integrable on $[a, b]$ and

$$
\widetilde{\int_{a}^{b}}(f \cdot g) \rho_{x}=\widetilde{\int_{a}^{b}} f \rho_{x} \cdot \widetilde{\int_{a}^{b}} g \rho_{x}
$$

Theorems 32, 33 and 34 will be used in section 4, where examples of integrable functions in the proportional sense will be given.

Theorem 28. If $f$ is positive and integrable on $[a, b]$, then for any number $\alpha$, the function $\alpha \odot f$ and $g$ is integrable on $[a, b]$ and

$$
\widetilde{\int_{a}^{b}} g \cdot(\alpha \odot f) \rho_{x}=\widetilde{\int_{a}^{b}} g \rho_{x} \cdot\left[\alpha \odot \widetilde{\int_{a}^{b}} f \rho_{x}\right] .
$$

Theorem 29. Suppose that $f$ is positive and integrable on $[a, b]$ and that $m \leq f(x) \leq M$, for all $x \in[a, b]$, then

$$
m \odot\left(\frac{b}{a}\right) \leq \widetilde{\int_{a}^{b}} f \rho_{x} \leq M \odot\left(\frac{b}{a}\right) .
$$

The last result can be used to prove the boundedness of solutions of proportional differential equations, see section 4 . 
Theorem 30. If $f$ is positive and proportionally integrable on $[a, b]$ and $F$ is defined on $[a, b]$ for

$$
F(x)=\widetilde{\int_{a}^{x}} f \rho_{x}
$$

then $F$ is continuous over $[a, b]$.

Theorem 31 (First Fundamental Theorem of the Proportional Calculus).

Let $f$ be a positive and proportionally integrable on $[a, b]$ and define $F$ on $[a, b]$ for

$$
F(x)=\widetilde{\int_{a}^{x}} f \rho_{x} .
$$

If $f$ is continuous in $c \in[a, b]$, then $F$ is differentiable at $c$, and

$$
\widetilde{F(c)}=f(c)
$$

Theorem 32 (Second Fundamental Theorem of the Proportional Calculus). If $f$ is a continuous positive function on $[a, b]$ and $f=\widetilde{h}$ for some function $h$, then

$$
\widetilde{\int_{a}^{b}} f \rho_{x}=\frac{h(b)}{h(a)}
$$

The last result can be used to solve proportional differential equations, see section 4 .

Proposition 33 (Proportional Integration by Parts). Let $u:[a, b] \rightarrow$ $\mathbf{R}^{+}$and $v:[a, b] \rightarrow \mathbf{R}^{+}$differentiable proportionally so the $u \odot v$ is integrable in the proportional sense. Then

$$
\widetilde{\int} u(x) \odot \widetilde{v(x)} \rho_{x}=\frac{u(x) \odot v(x)}{\widetilde{\int} v(x) \odot \widetilde{u(x)} \rho_{x}} .
$$

Proof. Let $u(x)$ and $v(x)$ two continuously proportional differentiable functions. The proportional product rule states

$$
\frac{\widetilde{d}}{d x}(u(x) \odot v(x))=v(x) \odot \frac{\tilde{d}}{d x} u(x) \cdot u(x) \odot \frac{\tilde{d}}{d x} v(x) .
$$

Integrating both sides with respect to $x$,

$$
\widetilde{\int} \frac{\widetilde{d}}{d x}(u(x) \odot v(x)) \rho_{x}=\widetilde{\int} v(x) \odot \frac{\widetilde{d}}{d x} u(x) \rho_{x} \cdot \widetilde{\int} u(x) \odot \frac{\widetilde{d}}{d x} v(x) \rho_{x} .
$$


Then, applying the definition of indefinite integral, we have

$$
\begin{gathered}
u(x) \odot v(x)=\widetilde{\int} v(x) \odot \frac{\widetilde{d}}{d x} u(x) \rho_{x} \cdot \widetilde{\int} u(x) \odot \frac{\widetilde{d}}{d x} v(x) \rho_{x}, \text { this equation is equivalent to } \\
\widetilde{\int} u(x) \odot \widetilde{v(x)} \rho_{x}=\frac{u(x) \odot v(x)}{\widetilde{\int} v(x) \odot \widetilde{u(x)} \rho_{x}} .
\end{gathered}
$$

This result gives the proportional version for the formula of integration by parts.

Example 34. The following equality holds

$$
\widetilde{\int} x \odot e^{x} \rho_{x}=\frac{x \odot e^{x}}{e^{x}} \cdot C,
$$

where $C$ is a positive constant. By using the substitution $u=x, \rho_{v}=e^{x} \rho_{x}$, equality $\widetilde{\int} e^{x} \rho_{x}=C \cdot e^{x}$ and the previous theorem we get the result.

We present some definitions that extend the usual integrable sense to the proportional integrable functions.

Definition 35 (Proportional Antiderivative). The function $F(x)$ is a Proportional Antiderivative of the function $f(x)$ on an interval $I$, if $\widetilde{F(x)}=$ $f(x)$ for all $x \in I$.

Definition 36 (Proportional Indefinite Integral). The proportional indefinite integral of $f(x)$ is the general proportional antiderivative of $f(x)$ this is expressed in the following terms

$$
\widetilde{\int} f(x) \odot \rho_{x}=\widetilde{\int} f(x) \rho_{x}=C \cdot F(x),
$$

where $C$ is a positive constant.

Example 37. The proportional indefinite integral of function $f(x)=x$ is

$$
\widetilde{\int} x \rho_{x}=C \cdot\left(x^{\{2\}}\right)^{\frac{1}{2}},
$$

where $C$ is a positive constant. Consider the function $F(x)=\left(x^{\{2\}}\right)^{\frac{1}{2}}$. It is easy to see that

$$
\left(\widetilde{x^{\{2\}}}\right)^{\frac{1}{2}}=[(\widetilde{x} \odot x) \cdot(x \odot \widetilde{x})]^{\frac{1}{2}}=\left((e \odot x) \cdot(x \odot e)^{\frac{1}{2}}=\left(x^{2}\right)^{\frac{1}{2}}=x\right.
$$


Example 38. We can deduce easily that

$$
\widetilde{\int} \cos _{e}(x) \rho_{x}=C \cdot \sin _{e}(x)
$$

as a consequence of Example 12, which states that $\widetilde{\sin _{e}(x)}=\cos _{e}(x)$.

Using the properties of the proportional derivation of section 3.1, the following propositions can be easily verified:

Proposition 39. Let $a, C$ and $K$ be positive constants. Then, we have

(a) $\tilde{\int} 1 \rho_{x}=K$.

(b) $\widetilde{\int} e^{n} \rho_{x}=C \cdot x^{n}$.

(c) $\tilde{\int} K \rho_{x}=C \cdot x \odot K$.

(d) $\widetilde{\int} e^{a \cdot x} \rho_{x}=C \cdot e^{a \cdot x}$.

(e) $\widetilde{\int} x^{\{2\}} \rho_{x}=C \cdot\left(x^{\{3\}}\right)^{\frac{1}{3}} \cdot$ (f) $\widetilde{\int} x^{\{n\}} \rho_{x}=C \cdot\left(x^{\{n+1\}}\right)^{\frac{1}{n+1}}$.

(g) $\widetilde{\int} e \oslash x \rho_{x}=\ln (x)$.

(h) $\widetilde{\int} e^{a \odot x} \rho_{x}=a^{\{-1\}} \odot e^{a \odot x}$.

(i) $\widetilde{\int} \frac{y(x)}{y(x)} \rho_{x}=\ln (y(x))$.

(j) $\int \sin _{e}(x) \rho_{x}=\frac{1}{\cos _{e}(x)}$.

\section{Applications}

\subsection{Ordinary Proportional Differential Equations}

In [33] the ordinary proportional differential equations are presented.

The simplest proportional differential equation to solve is

$$
\widetilde{x(t)}=f(t) \text {. }
$$

Solving this equation is equivalent to establishing the proportional integral of $f$. Using the fundamental theorem of the proportional calculus, the solution obtained is

$$
x(t)=C \cdot \widetilde{\int_{c}^{t}} f(s) \rho_{s},
$$

with $C$ a constant. 
Example 1. The solution of the proportional differential equation $\widetilde{x(t)}=$ $e^{t^{2}}$ is $x(t)=C \cdot\left(e^{t^{2}}\right)^{\frac{1}{2}}$. This is obtained deriving $C \cdot\left(e^{t^{2}}\right)^{\frac{1}{2}}$ applying the proportional chain rule. This result presents a contribution to the theoretical development, because unlike what happens in the theory of differential fields [35], $e^{t^{2}}$ admits a primitive in the context proportional.

Example 2. If $a(t)$ is a positive function and $x(c)=e^{K}$, then the solution of the proportional differential equation

$$
\widetilde{x(t)}=a(t) \odot x(t)
$$

is $x(t)=e^{K \cdot \widetilde{\int_{c}^{t}} a(s) \rho_{s}}$. This is obtained by representing the equation as $\widetilde{x(t) \oslash}$ $x(t)=a(t)$. Integrating proportionally of $c$ to $t$

$$
\widetilde{\int_{c}^{t}} \widetilde{x(s)} \oslash x(s) \rho_{s}=\widetilde{\int_{c}^{t}} a(s) \rho_{s} .
$$

By the fundamental theorem of proportional calculus we obtain $\frac{\ln (x(t))}{\ln (x(c))}=$ $\widetilde{\int_{c}^{t}} a(s) \rho_{s}$ and from the equality

$$
\ln (x(t))=\ln (x(c)) \cdot \widetilde{\int_{c}^{t}} a(s) \rho_{s}
$$

we obtain the solution. This equation will be useful to define the integrating factor.

Example 3. The Gompertz's function (See [39]).

$$
x(t)=a \cdot e^{-b \cdot e^{-c t}},
$$

can be linked with a proportional differential equation and it can be solved using the techniques presented in this work

Theorem 4. The Gompertz's function is solution of the following proportional differential equation

$$
\widetilde{x(t)}=\left[\frac{a}{x(t)}\right]^{c \cdot t}, x(1)=a \cdot e^{-\frac{b}{e^{c}}} .
$$


Proof. We are going to use the change of variable $y(t) \cdot x(t)=a$ for solving the equation for the unknown function $y(t)$. Proportionally deriving last equality, we have

$$
\widetilde{y(t)} \cdot \widetilde{x(t)}=1
$$

This equation can also be expressed as

$$
\widetilde{y(t)} \oslash y(t)=\frac{1}{e^{c \cdot t}} .
$$

Integrating proportionally the equation (4.3) we get

$$
\widetilde{\int_{1}^{t}} \widetilde{y(s)} \oslash y(s) \rho_{s}=\widetilde{\int_{1}^{t}} \frac{1}{e^{c \cdot s}} \rho_{s},
$$

by Theorem 32 we have

$$
\frac{\ln (y(t))}{\ln (y(1))}=\frac{\frac{1}{e^{c \cdot t}}}{\frac{1}{e^{c}}}
$$

Since $x(1)=a \cdot e^{-\frac{b}{e^{c}}}$ then $y(1)=e^{\frac{b}{e^{c}}}$ so $\ln (y(t))=b \cdot e^{-c \cdot t}$ which is equivalent to $y(t)=e^{b \cdot e^{-c \cdot t}}$. Finally, considering $x(t) \cdot y(t)=a$, we conclude that $x(t)=a \cdot e^{-b \cdot e^{-c t}}$, solution that corresponds Gompertz's function.

\subsubsection{Linear Proportional Differential Equations of first order}

Linear proportional differential equations of first order can be represented as the following way

$$
\widetilde{y} \cdot a(t) \odot y=b(t) .
$$

Consider $a(t)=a$ a positive constant, $b(t)=1$ and $\phi$ a solution of (4.4). Then we have

$$
\widetilde{\phi} \cdot a \odot \phi=1 .
$$

If we apply $e^{a \odot t}$ to both members of the equation the operation $\odot$, we obtain:

$$
e^{a \odot t} \odot(\widetilde{\phi} \cdot a \odot \phi)=1,
$$

or for Theorem 6 this equality is expressed

$$
\left(e^{a \odot t \odot \phi} \phi(t)\right)=1 \text {. }
$$


As a consequence of proposition 15 there is a constant $K$ such that $e^{a \odot t} \odot \phi(t)=K$, or

$$
\phi(t)=K \oslash e^{a \odot t} .
$$

It is possible to observe the analogies that this solution has with the solution of the exponential growth equation $y^{\prime}=a \cdot y$. We have shown that any solution $\phi$ of (4.4) must have the form (4.5), where $K$ is some constant. Conversely, if $K$ is any constant, the function $\phi$ defined by (4.5) is solution of (4.4). Indeed, we can use Theorem 11 to obtain the following equality:

$$
e^{\widetilde{\frac{1}{a \odot t}}}=\left(e^{t} \circ \frac{1}{a \odot t}\right) \odot\left(\frac{\widetilde{1}}{a \odot t}\right)
$$

where

$$
\frac{\widetilde{1}}{a \odot t}=\frac{\widetilde{1}}{a \odot t}=\frac{1}{(\widetilde{a} \odot t) \cdot(a \odot \widetilde{t})}=\frac{1}{a}
$$

Then

$$
e^{\frac{1}{a \odot t}}=e^{\frac{1}{a \odot t}} \odot\left(\frac{1}{a}\right) .
$$

Considering (4.5) and (4.6) we have

$$
\widetilde{\phi} \cdot a \odot \phi=\left(\widetilde{K^{\frac{1}{a \odot t}}}\right) a \odot K^{\frac{1}{a \odot t}}
$$

where

$$
\widetilde{K^{\frac{1}{a \odot t}}}=K \odot e^{\frac{1}{a \odot t}}=\left(\widetilde{K} \odot e^{\frac{1}{a \odot t}}\right)\left(K \odot e^{\widetilde{\frac{1}{a \odot t}}}\right)=1 \cdot K \odot e^{\widetilde{\frac{1}{a \odot t}}}
$$

Hence

$$
\widetilde{\phi} \cdot a \odot \phi=\left(K \odot e^{\frac{1}{a \odot t} \odot \frac{1}{a}}\right) \cdot a \odot K \odot e^{\frac{1}{a \odot t}}=\frac{1}{K \odot e^{\frac{1}{a \odot t} \odot a}}\left(a \odot K \odot e^{\frac{1}{a \odot t}}\right)=1 .
$$

We have proved the following theorem:

Theorem 5. Consider the proportional differential equation:

$$
\widetilde{y} \cdot a \odot y=1
$$

where $a$ is a positive constant. If $K$ is any number, the function $\phi$ defined by $\phi(t)=K^{\frac{1}{a \odot t}}$ is a solution of this equation, and moreover every solution has this form. 
Let $a$ be a positive constant and $b(t)$ a positive function defined on the interval $I$. Let the following proportional differential equation of first order

$$
\widetilde{y} \cdot a \odot y=b(t) .
$$

If $\phi$ is a solution of (4.9), then

$$
e^{a \odot t}(\widetilde{\phi} \cdot a \odot \phi)=e^{a \odot t} \odot b(t),
$$

by Theorem 6 , we have

$$
\left(e^{a \widetilde{\odot t} \odot \phi}\right)=e^{a \odot t} \odot b(t) .
$$

Let $u$ be a function such that

$$
\widetilde{u(t)}=e^{a \odot t} \odot b(t)
$$

that is

$$
u(t)=\int_{t_{0}}^{t} e^{a \odot s} \odot b(s) \rho_{s},
$$

where $t_{0}$ is some fixed point in $I$. Since $u$ and $e^{a \odot t} \phi(t)$ have the same derivative, it follows that

$$
e^{a \odot t} \odot \phi(t)=u(t) \cdot K,
$$

for some constant $K$. Therefore

$$
\phi(t)=(u(t) \cdot K) \oslash e^{a \odot t}=(u(t) \cdot K)^{\frac{1}{a \odot t}}=u(t)^{\frac{1}{a \odot t}} \cdot K^{\frac{1}{a \odot t}},
$$

then

$$
\phi(t)=u(t) \odot e^{\frac{1}{a \odot t}} \cdot K \odot e^{\frac{1}{a \odot t}} .
$$

Reciprocally, if $u$ is given by (4.10) and $\phi$ is defined by (4.11), where $K$ is any constant, then $\phi$ is solution of (4.9). This is verified as follows

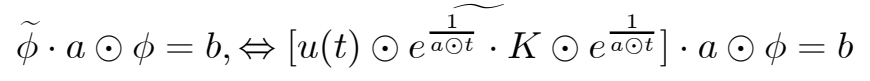




$$
\begin{aligned}
{\left[u(t) \odot e^{\frac{\widetilde{1}}{a \odot t}} \cdot K \odot e^{\frac{1}{a \odot t}}\right] } & =\left[\left(\widetilde{u(t)} \odot e^{\frac{1}{a \odot t}}\right) \cdot\left(u(t) \odot e^{\frac{1}{a \odot t}}\right)\right] \cdot\left[\left(\widetilde{K} \odot e^{\frac{1}{a \odot t}}\right) \cdot\left(K \odot e^{\frac{1}{a \odot t}}\right)\right] \\
& =\left[\left(\widetilde{u(t)} \odot e^{\frac{1}{a \odot t}}\right) \cdot\left(u(t) \odot e^{\frac{1}{a \odot t}} \odot \frac{1}{a}\right)\right]\left[K \odot e^{\frac{1}{a \odot t}} \odot \frac{1}{a}\right] .
\end{aligned}
$$

Then (4.11) follows:

$$
\begin{aligned}
& {\left[\left(\widetilde{u(t)} \odot e^{\frac{1}{a \odot t}}\right) \cdot\left(u(t) \odot e^{\frac{1}{a \odot t}} \odot \frac{1}{a}\right)\right]\left[K \odot e^{\frac{1}{a \odot t}} \odot \frac{1}{a}\right] \cdot a \odot \phi} \\
& =\left[\left(e^{a \odot t} \odot b \odot e^{\frac{1}{a \odot t}}\right)\left(\frac{1}{u(t) \odot e^{\frac{1}{a \odot t}} \odot a}\right)\right]\left[\frac{1}{K \odot e^{\frac{1}{a \odot t}} \odot a}\right] \cdot a \odot\left[u(t) \odot e^{\frac{1}{a \odot t}} \cdot K \odot e^{\frac{1}{a \odot t}}\right] \\
& =\left[\left(e^{a \odot t} \odot b \odot e^{\frac{1}{a \odot t}}\right)\left(\frac{1}{u(t) \odot e^{\frac{1}{a \odot t}} \odot a}\right)\right]\left(\frac{1}{K \odot e^{\frac{1}{a \odot t}} \odot a}\right)\left(u(t) \odot e^{\frac{1}{a \odot t}} \odot a\right)\left(K \odot e^{\left.\frac{1}{a \odot t} \odot a\right)}\right. \\
& =e^{a \odot t} \odot b \odot e^{\frac{1}{a \odot t}} \\
& =b \odot e^{a \odot t} \odot e^{\frac{1}{a \odot t}} \\
& =b \odot e=b \text {. }
\end{aligned}
$$

We summarize our result:

Theorem 6. Consider the proportional differential equation

$$
\widetilde{y} \cdot a \odot y=b(t),
$$

where $a$ is a positive constant, $b(t)$ is a continuous function defined on an interval $I$ and $K$ is any positive constant. The solution to this equation is

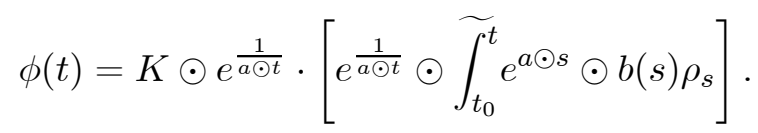

To solve the linear proportional differential equation when $a(t)$ and $b(t)$ are positive functions, we proceed analogously considering $u(t)=e^{\widetilde{\int} a(s) \rho_{s}}$ as an integrating factor, we obtain the following result:

Theorem 7. The unique solution of $\widetilde{x(t)} \cdot a(t) \odot x(t)=b(t)$, considering $x\left(t_{0}\right)=x_{0}$ is given by the following representation

$$
\left.x(t)=\left[\left(x_{0}\right) \odot u_{0}\right) \oslash u(t)\right] \cdot\left[\left(\widetilde{\int_{t_{0}}^{t}} b(s) \odot u(s) \rho_{s}\right) \oslash u(t)\right],
$$

where $u(t)$ is the solution of the proportional linear homogeneous equation. 
On the other hand, the problem of finding bounded solutions of first- order linear differential equations, in its classical version, was solved by Perron in 1930 (see [32]). In the proportional context, considering equation (4.9), we have:

- If $a=1$. In the case $b(t) \equiv 1$ no uniqueness is obtained. In the case $b(t) \equiv e$ no existence is obtained.

- If $a>1$, the bounded solution of (4.9) is:

$$
\phi_{p_{0}}(t)=\widetilde{\int_{0}^{t}} e^{a^{-1} \odot\left(\frac{t}{s}\right)} \odot b(s) \rho_{s} .
$$

- If $0<a<1$, the bounded solution of (4.9) is:

$$
\phi_{p_{\infty}}(t)=\left[\widetilde{\int_{t}^{\infty}} e^{a^{-1} \odot\left(\frac{t}{s}\right)} \odot b(s) \rho_{s}\right]^{-1} .
$$

Next, we define a type of bounded functions of a proportional nature.

Definition 8 ( $q$-Periodic Function). Let $f$ be a positive function, it is called a $q$-periodic function if there exists $q \in \mathbf{R}^{+}$such that

$$
f(t)=f(t \cdot q) \text { for all } t \in \mathbf{R}^{+} .
$$

Example 9. The function $g(t)=e^{\cos (\ln (t))}=\cos _{e}(t)$ is $e^{2 \cdot k \cdot \pi}-$ periodic for any integer number $k$. The representative period is $e^{2 \cdot \pi}$. In example 11, we obtained $\widetilde{\cos _{e}}(t)=\frac{1}{\operatorname{sen}_{e}(t)}$.

Example 10. The function $f(t)=e^{\sin (\ln (t))}=\sin _{e}(t)$ is $e^{2 \cdot k \cdot \pi}-$ periodic for any integer number $k$. The representative period is $e^{2 \cdot \pi}$. In example 12, we obtained $\sin _{e}(t)=\cos _{e}(t)$.

Figure 4.1 shows other examples of this type of oscillatory functions. From the above we present the following result 


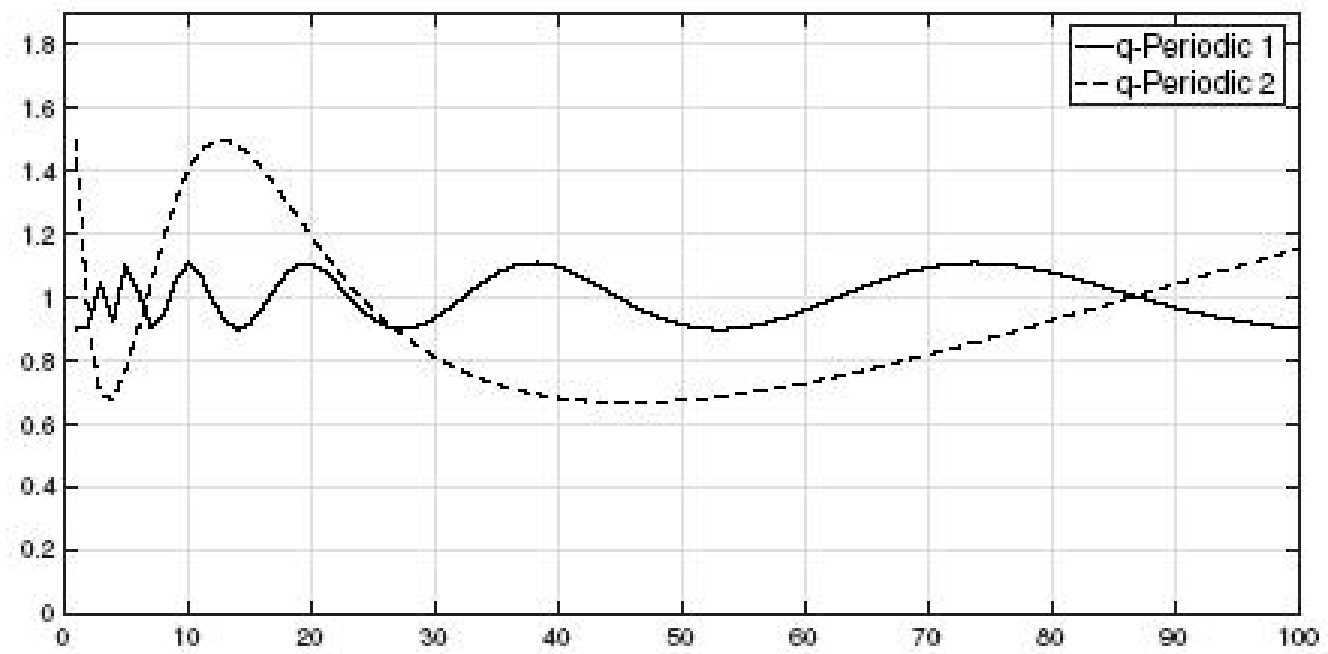

Figure 4.1: Example of q-Periodic functions. The q-Periodic 1, is $f(t)=(0.9)^{\cos \left(\log _{0.9}(t)\right)}$, has a representative period $(0.9)^{2 \cdot \pi}$. The q-Periodic 2 , is $g(t)=(1.5)^{\sin \left(\log _{1.5}(t)\right)}$, has a representative period $(1.5)^{2 \cdot \pi}$.

Theorem 11. If in (4.9), the function $b(t)$ is a $q$-periodic function, then the particular solution $\phi_{p_{0}}(t)$ is also a $q$-periodic function.

Proof. The following bounded solution is considered $\phi_{p_{0}}(t)=\widetilde{\int_{0}^{t}} e^{a^{-1} \odot\left(\frac{t}{s}\right)} \odot$ $b(s) \rho_{s}$. Making the change of variable $s=u \cdot q$, result $\rho_{s}=\rho_{u}$ and as $b(u \cdot q)=b(u)$ the following equality is obtained

$$
\phi_{p_{0}}(t \cdot q)=\widetilde{\int_{0}^{t \cdot q}} e^{a^{-1} \odot\left(\frac{t \cdot q}{s}\right)} \odot b(s) \rho_{s}=\widetilde{\int_{0}^{t}} e^{a^{-1} \odot\left(\frac{t}{u}\right)} \odot b(u) \rho_{u}=\phi_{p_{0}}(t) .
$$

From this equality, we obtain that $\phi_{p_{0}}$ is $q$-periodic.

Example 12. Let the following nonlinear proportional differential equation of first order

$$
\widetilde{y(t)} \cdot[p(t) \odot y(t)]=q(t) \odot y(t)^{\{n\}}, n \in \mathbf{N}^{*}, n>2 .
$$


This equation is the so-called Proportional Bernoulli Equation. Using the change of variable $z=e \oslash y^{\{n-1\}}$, we obtain the following nonhomogeneous linear equation of first order

$$
\widetilde{z(t)} \cdot p(t) \odot z(t)=q(t)^{\{1-n\}},
$$

and it can be easily solved.

Remark 13. When $n=3$ this equation corresponds to the proportional logistic equation which will be studied in the last section.

Example 14. Let the following nonlinear proportional differential equation of first order

$$
\widetilde{y(t)}=\frac{1}{q(t)} \odot y(t)^{\{2\}} \cdot \frac{1}{p(t)} \odot y(t) \cdot r(t) .
$$

This equation is the so-called the proportional Riccati equation. Using the change of variable $y=y_{1} \cdot(e \oslash z)$, where $y_{1}$ is a known solution of the proportional Riccati equation, we can obtain the following non-homogeneous equation of first order,

$$
\widetilde{z(t)}=\left[p(t) \cdot\left(q(t) \odot y_{1}\right)^{2}\right] \cdot \frac{1}{q(t)} \odot z(t)^{\{2\}} .
$$

To conclude this section, we present the useful Gronwall lemma in its proportional version, which can be used for non-negative functions to prove stability and uniqueness of solutions.

Theorem 15 (Proportional-Gronwall Inequality). Let $K \geq 1$ constant and let $f, g \geq 1$ be continuous functions on some interval $a \leq t \leq b$ satisfying the inequality

$$
f(t) \leq K \cdot \widetilde{\int_{a}^{t}} f(s) \odot g(s) \rho_{s}, \text { for } a \leq t \leq b .
$$

Then

$$
f(t) \leq K \odot \exp \left(\widetilde{\int_{a}^{b}} g(s) \rho_{s}\right), \text { for } a \leq t \leq b .
$$


Proof. Let $R(t)=K \cdot \widetilde{\int}_{a}^{t} f(s) \odot g(s) \rho_{s}$. Then $f(t) \leq R(t)$ by hypothesis. Because $g(t) \geq 1$, we obtain $f(t) \odot g(t) \leq R(t) \odot g(t)$, we have $\widetilde{R(t)}=$ $f(t) \odot g(t)$. Therefore $R(a)=K$ and

$$
\widetilde{R(t)} \leq R(t) \odot g(t)
$$

Considering the integrating factor $\exp \left(\left[\widetilde{\int_{a}^{t}} g(s) \rho_{s}\right]^{-1}\right)$, and using $\odot$, we obtain

$$
\frac{\widetilde{R(t)} \odot \exp \left(\left[\widetilde{\int_{a}^{t}} g(s) \rho_{s}\right]^{-1}\right)}{R(t) \odot g(t) \odot \exp \left(\left[\widetilde{\int_{a}^{t}} g(s) \rho_{s}\right]^{-1}\right)} \leq 1
$$

or

$$
\left.R(t) \odot \exp \left(\widetilde{\left[\tilde{\int}_{a}^{t}\right.} g(s) \rho_{s}\right]^{-1}\right) \leq 1 .
$$

Integrating using the proportional integral from $a$ a $t$ gives

$$
\frac{R(t) \odot \exp \left(\left[\widetilde{\int_{a}^{t}} g(s) \rho_{s}\right]^{-1}\right)}{R(a)} \leq 1,
$$

since $f(t) \leq R(t)$ and $R(a)=K$,

$$
f(t) \leq K \odot \exp \left(\widetilde{\int_{a}^{b}} g(s) \rho_{s}\right), \text { for } a \leq t \leq b .
$$

which is the desired inequality.

\subsubsection{Linear Proportional Differential Equation of second order}

Linear proportional differential equation of second order has the form

$$
\widetilde{\widetilde{y}} \cdot(P(t) \odot \widetilde{y}) \cdot Q(t) \odot y=R(t),
$$

where $P, Q$, and $R$ are positive and continuous functions. The case where $R(t)=1$ is called homogeneous linear equation of second order, expressed as 


$$
\widetilde{\widetilde{y}} \cdot(P(t) \odot \widetilde{y}) \cdot Q(t) \odot y=1,
$$

If $R(t)=1$ for some $t$, then (4.20) is called non-homogeneous. If we know two solutions $\phi_{1}$ and $\phi_{2}$ of (4.21), then the linear combination $c_{1} \odot \phi_{1} \cdot c_{2} \odot \phi_{2}(t)$, is also a solution of (4.21), so this can be stated as follows:

Theorem 16. If $\phi_{1}(t)$ and $\phi_{2}(t)$ are solutions of (4.21), then

$$
c_{1} \odot \phi_{1} \cdot c_{2} \odot \phi_{2}(t),
$$

is also a solution of (4.21) for all $c_{1}, c_{2}$ positive constants.

In general, it is not easy to find a particular solution of linear proportional differential equation of second order. But it is always possible to do it so if the coefficient functions $P, Q, R$ are constant functions. That is, if the proportional differential equation has the form

$$
\widetilde{\widetilde{y}} \cdot(P \odot \widetilde{y}) \cdot Q \odot y=R .
$$

For the case $P=e, Q=e, R=1$ and $y(1)=1, \widetilde{y(1)}=e$, the equation $\widetilde{\widetilde{y}} \odot y=1$ has as solutions

$$
y_{1}(t)=\sin _{e}(t), \quad y_{2}(t)=\cos _{e}(t) .
$$

This was presented before in Examples 4 and 5. The general solution for this equation can be expressed as

$$
y(t)=c_{1} \odot \sin _{e}(t) \cdot c_{1} \odot \cos _{e}(a \odot t),
$$

for any $c_{1}, c_{2}$,positive constants. The following result presents a characterization of the general solution of (4.20).

Theorem 17. Let $\phi_{g}$ be a general solution of equation $\underset{\widetilde{y}}{\widetilde{y}} P(t) \odot \widetilde{y} \cdot Q(t) \odot$ $y=1$ and let $\phi_{p}$ be a particular solution of the equation $\widetilde{\widetilde{y}} \odot P(t) \odot \widetilde{y} \cdot Q(t) \odot$ $y=R(t)$. Then $\phi_{g} \cdot \phi_{p}$ is a general solution of $\widetilde{\widetilde{y}} \odot P(t) \odot \widetilde{y} \cdot Q(t) \odot y=R(t)$. 


\subsection{Proportional Heat and Wave Equations}

In this section we are interested in the study of functions of two variables, $w: \mathbf{R}^{+} \times \mathbf{R}^{+} \rightarrow \mathbf{R}^{+}$, which can be expressed as

$$
w(x, t)=u(x) \odot v(t),
$$

where $u$ and $v$ are positive functions. The partial proportional derivative of $w(x, t)$ with respect to $x$ at the point $(a, b)$ works in the same way as in the proportional derivative of the function $g(x)=w(x, b)$, since $\frac{\partial w}{\partial x}(a, b)=\widetilde{g(a)}$. So we conclude that

$$
\frac{\widetilde{\partial w}}{\partial x}(a, b)=\lim _{h \rightarrow 1}\left(\frac{f(a \cdot h, b)}{f(a, b)}\right) \oslash h .
$$

In a similar way, we define

$$
\frac{\widetilde{\partial w}}{\partial t}(a, b)=\lim _{h \rightarrow 1}\left(\frac{f(a, b \cdot h)}{f(a, b)}\right) \oslash h .
$$

Considering these definitions it is possible to express the proportional heat equation:

$$
a^{\{2\}} \odot \frac{\widetilde{\partial^{2}} w}{\widetilde{\partial} x^{2}}=\frac{\widetilde{\partial} w}{\widetilde{\partial} t},
$$

which in additive terms has been extensively applied to several natural phenomenon.

Theorem 18. Consider the proportional heat equation

$$
a^{\{2\}} \odot \frac{\widetilde{\partial^{2}} w}{\widetilde{\partial} x^{2}}=\frac{\widetilde{\partial} w}{\widetilde{\partial} t},
$$

where the function $w: \mathbf{R}^{+} \times \mathbf{R}^{+} \rightarrow \mathbf{R}^{+}$satisfies $w(1, t)=1$ and $w\left(e^{\pi}, t\right)=$ 1. Then a solution of heat is

$$
w_{n}(x, t)=e^{\frac{1}{n^{22\}} \odot a^{\{2\}} \odot t} \odot \sin _{e}(n \odot x) .}
$$


Proof. Using solutions of separable variables type $w(x, t)=u(x) \odot v(t)$ in heat, we obtain two proportional differential equations

$$
\widetilde{\widetilde{u(x)}} \oslash u(x)=\frac{1}{\lambda}
$$

and

$$
\left.\left(e \oslash a^{\{2\}}\right) \odot \widetilde{(v(t)} \oslash v(t)\right)=\frac{1}{\lambda} .
$$

Equation first is equivalent to $\widetilde{\widetilde{u(x)}} \cdot \lambda \odot u=1$. For the cases $\lambda>1$ and $\sqrt{\lambda_{e}}:=e^{\sqrt{\ln (\lambda)}}$, this equation has as general solution:

$$
u(x)=C_{1} \odot \operatorname{sen}_{e}\left(\sqrt{\lambda_{e}} \odot x\right) \cdot C_{2} \odot \cos \left(\sqrt{\lambda_{e}} \odot x\right) .
$$

Considering the boundary conditions $w(1, t)=u(1)=1$, it follows that $C_{2}=1$. Then $u$ is reduced to $y(x)=C_{1} \odot \operatorname{sen}_{e}\left(\sqrt{\lambda_{e}} \odot x\right)$, without loss of generality we will assume that $C_{1}=e$. To satisfy the second contour condition $w\left(e^{\pi}, 1\right)=u\left(e^{\pi}\right)=1$, it must be satisfied that $1=\sin _{e}\left(\sqrt{\lambda_{e}} \odot e^{\pi}\right)$, which is equivalent to $\ln (\lambda)=n^{2}$, therefore we get that $\lambda=e^{n^{2}}$. We conclude that

$$
u(x)=\sin _{e}\left(e^{n} \odot x\right) .
$$

$v(t)=e^{\frac{1}{n^{\{2\}} \odot a^{22} \odot t}}$ is the obvious solution of the second equation. Resulting products of the form $w_{n}(x, t)=e^{\frac{1}{n^{\{2\}} \odot a^{\{2\}} \odot t}} \odot \sin _{e}(n \odot x)$, which are solutions of the proportional heat equation.

Remark 19. A finite proportional linear combination of the $w_{n}(x, t)$,

$$
b_{1} \odot w_{1} \cdot b_{2} \odot w_{2} \cdot b_{3} \odot w_{3} \cdots b_{n} \odot w_{n},
$$

is also the solution of the equation (46).

Remark 20. If we do not consider the convergence and differentiability of the terms, we have that the following infinity product is a solution

$$
w(x, t)=\prod_{n=1}^{\infty} b_{n} \odot e^{\frac{1}{\left(e^{n}\right)^{\{2\}} \odot\left(e^{a}\right)^{\{2\}} \odot t}} \odot \sin _{e}\left(e^{n} \odot x\right) .
$$

The classical theory of Fourier Series discussed by several mathematicians in the mid-eighteenth century, arises naturally when we study the vibrations of a string. This study leads to the solvability of the well-known equation of the vibrating string, expressed classically as: 


$$
a^{2} \cdot \frac{\partial^{2} w}{\partial^{2} x^{2}}=\frac{\partial^{2} w}{\partial t^{2}}
$$

In the proportional context, proceeding in a similar way to Theorem 18, we obtain the following result:

Theorem 21. Consider the proportional wave equation

$$
a^{\{2\}} \odot \frac{\widetilde{\partial^{2}} w}{\widetilde{\partial} x^{2}}=\frac{\widetilde{\partial^{2}} w}{\widetilde{\partial} t^{2}} .
$$

If the function $w: \mathbf{R}^{+} \times \mathbf{R}^{+} \rightarrow \mathbf{R}^{+}$satisfies $w(1, t)=1, w\left(e^{\pi}, t\right)=1$ and $\frac{\partial w(\widetilde{x, 0)}}{\partial t}=1$ then a solution of cuerda is

$$
w_{n}(x, t)=\operatorname{sen}_{e}\left(e^{n} \odot x\right) \odot \cos _{e}\left(e^{n} \odot e^{a} \odot t\right) .
$$

It is possible to perform observations analogous to 19 and 20 referred to the proportional wave equation solution.

\subsection{Proportional Fourier Series}

The infinite products presented in the previous section allow us to formalize what we understand by proportional Fourier series in the context of proportional arithmetic. The classical representation of the Fourier Series has been applied in several areas such as acoustics, optics, electrical engineering, vibration analysis, signal processing and quantum mechanics. In this section we present the proportional Fourier series constructed by using the $q$-Periodic Functions, presented in Definition 8.

Definition 22. The proportional Fourier series of a function $f(x)$ with period $e^{2 \pi}$ is given by

$$
\prod_{k=1}^{\infty}\left[a_{k} \odot \cos _{e}(k \odot x)\right] \cdot\left[b_{k} \odot \sin _{e}(k \odot x)\right] .
$$

Where

$$
\begin{aligned}
& a_{1}=e^{\frac{1}{2 \pi}} \widetilde{\int_{e^{\frac{1}{\pi}}}^{e^{\pi}}} f(x) \rho x \\
& b_{1}=1
\end{aligned}
$$




$$
\begin{aligned}
& a_{k}=e^{\frac{1}{\pi} \int_{e^{\frac{1}{\pi}}}^{e^{\pi}}} f(x) \odot \cos _{e}(k \odot x), \\
& b_{k}=e^{\frac{1}{\pi} \int_{e^{\frac{1}{\pi}}}^{e^{\pi}}} f(x) \odot \sin _{e}(k \odot x) .
\end{aligned}
$$

These are the coefficients of the proportional Fourier Series. In analogy to the proportional Taylor series, we define the proportional Fourier polynomials to be the finite sum:

$$
W_{n}=\prod_{k=1}^{n}\left[a_{k} \odot \cos _{e}(k \odot x)\right] \cdot\left[b_{k} \odot \sin _{e}(k \odot x)\right] .
$$

It is possible to extend these studies by defining the proportional Fourier transform and complete the theory to introduce new tools for mathematical modeling.

\section{Non-Newtonian Logistic Growth Model}

The Ordinary Logistic Equation describes a type of growth. The first model was presented by Verhulst, who derived his logistic equation to describe the self-limiting growth of a biological population (see [31]). This type of growth has been studied from different perspectives it has been generalized in several works (see [4, 27]). In [34] the proportional logistic equation was proposed and studied in terms of proportional arithmetic. Let $N$ be the population size and $t$ the time. This model is formalized by the following proportional differential equation:

$$
\tilde{N}=r \odot N \odot\left(\frac{e}{N \oslash K}\right) .
$$

Where $\widetilde{N}$ represents the proportional rate of population change, $r=$ 1 the proportional growth rate and $K$ the environmental capacity or carrying capacity of the environment [14]. When $N$ is close to 1 , the equation takes the form of exponential growth:

$$
\widetilde{N}=r \odot N,
$$

and the $\left(\frac{e}{N \oslash K}\right)$ factor represents the limited growth of the population. For the case $N=K$, the proportional differential equation (5.1) corresponds to

$$
\widetilde{N}=1
$$


and it represents the idea of no variation in the proportional calculus context. To find the equilibrium solutions, we must solve

$$
1=r \odot N \odot\left(\frac{e}{N \oslash K}\right) .
$$

Since $r=1$, we have

$$
N=1 \text { or } \frac{e}{N \oslash K}=1,
$$

so we conclude that equilibria solutions are $N=1$ and $N=K$. The following result shows that by means of analytical techniques exclusively from proportional arithmetic it is possible to obtain the solution of this equation.

Theorem 1. Considering the initial condition $N(1)=N_{1}$, the solution of equation (5.1) is

$$
N(t)=\left(N_{1} \odot K\right) \oslash\left[\left(\frac{K}{N_{1}}\right) \odot e^{\frac{1}{r \odot t}} \cdot N_{1}\right] .
$$

Proof. To solve equation (5.1), we will rewrite it in an equivalent form

$$
e \oslash\left[N \odot\left(\frac{e}{N \oslash K}\right)\right] \odot \widetilde{N}=r .
$$

To obtain the solution, we present the following steps:

Step one: Let $A, B$ constants such that

$$
e \oslash\left[N \odot\left(\frac{e}{N \oslash K}\right)\right]=(A \oslash N) \cdot\left(B \oslash\left[\left(\frac{e}{N \oslash K}\right)\right]\right) .
$$

In order to find the constants $A$ and $B$, we use the property:

$$
(a \oslash x) \cdot(b \oslash y)=(a \odot x) \cdot(b \odot y) \oslash(x \odot y)
$$

We conclude that the left side of the equation (5.6) equals to

$$
(A \odot N) \cdot\left(B \odot\left(\frac{e}{N \oslash K}\right)\right) \oslash\left(N \odot\left(\frac{e}{N \oslash K}\right)\right) .
$$


Step two: Factoring the expression

$$
(A \odot N) \cdot\left(B \odot\left(\frac{e}{N \oslash K}\right)\right)=A \cdot\left(N \odot\left(\frac{B}{A \oslash K}\right)\right),
$$

and equalizing coefficients of the equations (5.6) we get

$$
A \cdot\left(N \odot\left(\frac{B}{A \oslash K}\right)\right)=e=e \cdot(1 \odot N) .
$$

Hence, we conclude that $A=e$ and $\frac{B}{A \oslash K}=1$, thus $B=e \oslash K$. We have deduced that equation (5.1) is equivalent to

$$
(e \oslash N) \cdot\left((e \oslash K) \oslash\left(\frac{e}{N \oslash K}\right)\right) \odot \widetilde{N}=r .
$$

Step three: To integrate proportionally (5.7), we will consider the following equalities

- $\operatorname{ln(N)}=e \oslash N$, differentiating with respect to $N$.

- $\widetilde{\ln (N)}=(e \oslash N) \odot \widetilde{N}$, differentiating with respect to $t$.

- $\ln \left(\frac{e}{N \oslash K}\right)=(e \oslash K)^{-1} \odot\left[e \oslash\left\{\frac{e}{N \oslash K}\right\}\right]$, differentiating with respect to $N$.

- $\ln \left(\widetilde{e} \frac{e}{N \oslash K}\right)=(e \oslash K)^{-1} \odot\left[e \oslash\left\{\frac{e}{N \oslash K}\right\}\right] \odot \tilde{N}$, differentiating with respect to $t$.

Step four: Consider equation (5.7) and the property $\left(a^{-1} \oslash b\right)^{-1}=a \oslash b$. We have

$$
(e \oslash N) \odot \tilde{N} \cdot\left\{\left((e \oslash K)^{-1} \oslash\left(\frac{e}{N \oslash K}\right)\right) \odot \tilde{N}\right\}^{-1}=r .
$$

Step five: Integrating proportionally with respect to time, we obtain that equation (5.7) is equivalent to

$$
(\ln (N)) \cdot\left(\ln \left(\frac{e}{N \oslash K}\right)\right)^{-1} \cdot R_{1}=(r \odot t) \cdot R_{2},
$$


where $R_{1}, R_{2}$ are constants of proportional integration (notice that $1<$ $N<K)$. Considering that $C_{1}=\frac{R_{2}}{R_{1}}$ and the property $\frac{\ln (a)}{\ln (b)}=\ln (a \oslash b)$, equation (5.9) becomes to

$$
\ln \left(N \oslash\left(\frac{e}{N \oslash K}\right)\right)=(r \odot t) \cdot C_{1},
$$

which is equivalent to

$$
e^{(r \odot t) \cdot C_{1}}=N \oslash\left(\frac{e}{N \oslash K}\right) .
$$

Defining $C_{1}=\ln \left(C_{2}\right)$, we have

$$
C_{2} \odot e^{r \odot t}=N \oslash\left(\frac{e}{N \oslash K}\right) .
$$

Step six: The equation (5.11) is equivalent a to the algebraic relation

$$
C_{3} \odot e^{(r \odot t)^{-1}}=\left(\frac{e}{N \oslash K}\right) \oslash N
$$

In order to prove the proposition, we will use the following fact: If $c_{1} \odot$ $e^{x}=b \oslash a$, then there exists $c_{2}$ such that $c_{2} \odot e^{x^{-1}}=a \oslash b$. Hence, using $\left(\frac{a}{b}\right) \oslash c=\frac{a \oslash c}{b \oslash c}$ equation (5.11) can be expressed as

$$
\frac{(e \oslash N)}{(e \oslash K)}=C_{3} \odot e^{\frac{1}{r \odot t}}
$$

Step seven: From equation (5.12) we conclude that

$$
e \oslash N=\left(C_{3}\right) \odot e^{\frac{1}{r \odot t}} \cdot(e \oslash K) .
$$

Also, we have the following analytical expression for a solution of equation

$$
N(t)=e \oslash\left[\left(C_{3} \odot e^{\frac{1}{r \odot t}}\right) \cdot(e \oslash K)\right]
$$

Step eight: To find the constant $C_{3}$, we consider the initial condition $N_{1}$ and we solve the following equation

$$
N_{1}=e \oslash\left[\left(C_{3} \odot e\right) \cdot(e \oslash K)\right],
$$


where the solution is:

$$
C_{3}=\frac{e \oslash N_{1}}{e \oslash K}
$$

Step nine: We conclude that the solution of equation (5.1) is

$$
N(t)=e \oslash\left[\left(\frac{e \oslash N_{1}}{e \oslash K}\right) \odot e^{\frac{1}{r \odot t}} \cdot(e \oslash K)\right] .
$$

Step ten: Finally, we perform an algebraic arrangement, exponentiating by $N_{1} \odot K$, the solution to equation (5.1) is

$$
N(t)=\left(N_{1} \odot K\right) \oslash\left[\left(\frac{K}{N_{1}}\right) \odot e^{\frac{1}{r \odot t}} \cdot N_{1}\right] .
$$

We recall that the solution of the traditional logistic equation is:

$$
N(t)=\frac{K \cdot N_{0}}{\left(K-N_{0}\right) e^{-r t}+N_{0}} .
$$

It is important to remark the analogies of these two equations that represent a type of logistic growth. As an example of a solution, we consider $N_{1}=e, K=e^{2}$ and $r=e$. For these values the solution of the equation is:

$$
N(t)=e^{\frac{2 t}{1+t}} .
$$

Finally, a brief stability study is carried out, it is recalled that an equilibrium solution $N=c$ is called stable if any solution $N(t)$ that starts near $N=c$ stays near $c$. The equilibrium $N=c$ is called asymptotically stable if any solution $N(t)$ that starts near $N=c$ actually converges to $c$, that is

$$
\lim _{t \rightarrow \infty} N(t)=c .
$$

Notice that, in the example, the equilibrium solution $N=K$ is asymptotically stable, due to

$$
\lim _{t \rightarrow \infty} N(t)=K=e^{2} \approx 7.38905609893 .
$$




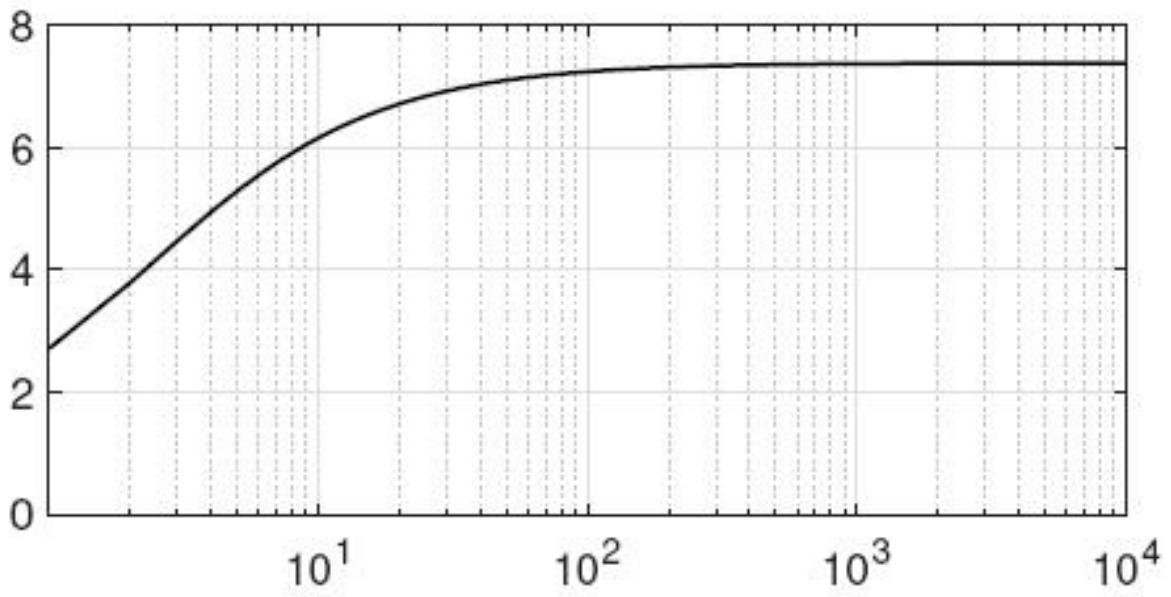

Figure 5.1: Solution of the proportional logistic equation. The $t$ axis is considered in logarithmic scale. This image allows us to observe asymptotic behaviour.

\section{Acknowledgements}

William Campillay-Llanos thanks the funding granted by the Doctoral Scholarship of Universidad Católica del Maule. Felipe Guevara-Morales thanks for the support of DIUDA scientific initiation project 22372. Manuel Pinto and Ricardo Torres thank for the support of Fondecyt projects 1120709 and 1170466 . 


\section{References}

[1] T. M. Apostol, Análisis matemático, 2nd ed. Barcelona: Reverté, 1996.

[2] A. E. Bashirov, E. M. Kurpinar, and A. Özyapıcl, "Multiplicative calculus and its applications", Journal of mathematical analysis and applications, vol. 337, no. 1, pp. 36-48, Jan. 2008, doi: 10.1016/j.jmaa.2007.03.081

[3] A. E. Bashirov and M. Riza, "On complex multiplicative differentiation", TWMS Journal of applied and engineering mathematics, vol. 1, no. 1, pp. 75-85, 2011. [On line]. Available: https:/ / bit.ly/ 34HcPb6

[4] C. Birch, "A new generalized logistic sigmoid growth equation compared with the Richards growth equation", Annals of botany, vol. 83, no. 6, pp. 713-723, Jun. 1999, doi: 10.1006/ anbo.1999.0877

[5] K. Boruah and B. Hazarika, "Application of geometric calculus in numerical analysis and difference sequence spaces", Journal of mathematical analysis and applications, vol. 449, no. 2, pp. 1265-1285, May 2017, doi: 10.1016/j.jmaa.2016.12.066

[6] J. P. Ramis, A. Warusfel, and F. Moulin, Dirs., Cours de Mathématiques pures et appliquées: algèbre et géométrie, vol. 1. Bruxelles: De Boek, 2010.

[7] M. S. Burgin, "Non-diophantine arithmetics or is it possible that 22 is not equal to 4?", Home Page for Markin Burgin - UCLA Library. [Online]. Available: https:/ / bit.ly/ 3ec0TkJ

[8] M. Burgin, "Nonclassical models of the natural numbers", Uspekhi matematicheskikh nauk, vol. 32, no. 6, pp. 209-210, 1977. [On line]. Available: https:/ / bit.ly/2GbY46D

[9] K. Boruah and B. Hazarika, "On some generalized geometric difference sequence spaces”, Proyecciones (Antofagasta. On line), vol. 36, no. 3, pp. 373-395, Sep. 2017, doi: 10.4067/ S0716-09172017000300373 
[10] W. Campillay-Llanos, "Cálculo proporcional: un maravilloso recorrido por el mundo multiplicativo", Undergraduate Thesis, Universidad Metropolitana de Ciencias de la Educación, 2007. [On line]. Available: https:/ / bit.ly/ 2TG1gL8

[11] W. Campillay-Llanos and F. Guevara-Morales, "Proportional algebraic curves, Geometry session," in XXXI Jornada matemática de la zona sur, Universidad Austral de Chile, 25, 26 y 27 de abril de 2018, Valdivia, Chile. [On line]. Available: https:/ / bit.ly/ 3edxt5D

[12] W. Campillay-Llanos, F. Guevara-Morales, "Gelfond's constant and proportional arithmetic", in XXVIII Jornada matemática de la zona sur, Universidad del Bío-Bío, 22 al 24 de abril, Chillán, Chile, 2015. [On line]. Available: https:/ / bit.ly/ 3ebuj2j

[13] W. Campillay-Llanos, F. Guevara-Morales, M. Pinto, and R. Torres, "Differential and integral proportional calculus: how to find a primitive for Gaussian function", International journal of mathematical education in science and technology, pp. 1-14, 2020, doi: 10.1080/ 0020739X.2020.1763489

[14] E. J. Chapman and C. J. Byron, "The flexible application of carrying capacity in ecology", Global ecology and conservation, vol. 13, Art ID. e00365, Jan. 201, doi: 10.1016/ j.gecco.2017.e00365

[15] F. Córdova-Lepe, "The multiplicative derivative as a measure of elasticity in economics", TMAT Revista latinoamericana de ciencias e ingeniería, vol. 2, no. 3, pp. 1-8, Jan. 2006. [On line]. Available: https:/ / bit.ly/ 3jGALzB

[16] F. Córdova-Lepe and M. Pinto, "From quotient operation toward a proportional calculus", International journal of mathematics, game theory and algebra, vol. 18, no. 6, pp. 527-536, 2009.

[17] M. Czachor, "Relativity of arithmetic as a fundamental symmetry of physics", Quantum studies: mathematics and foundations, vol. 3, no. 2, pp. 123-133, Jun. 2016, doi: 10.1007/ s40509-015-0056-4

[18] M. Czachor, "If gravity is geometry, is dark energy just arithmetic?", International journal of theoretical physics, vol. 56, no 4, pp. 1364-1381, Apr. 2017, doi: 10.1007/ s10773-017-3278-x

[19] J. P. DeLong and D. A. Vasseur, "A dynamic explanation of size-density scaling in carnivores", Ecology, vol. 93, no. 3, pp. 470-476, Mar. 2012, doi: 10.1890/11-1138.1 
[20] O. Dovgoshey, O. Martio, V. Ryazanov, and M. Vuorinen, "The cantor function", Expositiones mathematicae, vol. 24, no. 1, pp. 1-37, Feb. 2006, doi: 10.1016/ j.exmath.2005.05.002

[21] R. Katz and M. Grossman, Non-Newtonian calculus. Pigeon Cove, MA: Lee Press, 1972.

[22] M. Grossman, Bigeometric calculus: a system with a scale-free derivative, Rockport, MA: Archimedes Foundation, 1983.

[23] M. Grossman, The first nonlinear system of differential and integral calculus, Rockport, MA: Mathco, 1979.

[24] G. M. N' Guerekata, Ed., New Research on Evolution Equations, New York. NY: Nova Science Publishers, 2010.

[25] J. Hofbauer and K. Sigmund, The theory of evolution and dynamical systems: mathematical aspects of selection, Cambridge: Cambridge University Press, 1988.

[26] J. Kirkwood, Mathematical physics with partial differential equations, 2nd ed. London: Academic Press, 2018.

[27] B. Kooi, M. Boer, and S. Kooijman, "On the use of the logistic equation in models of food chains", Bulletin of mathematical biology, vol. 60, no. 2, pp. 231-246, 1998, doi: 10.1006/ bulm.1997.0016

[28] D. Laugwitz, "On the historical development of infinitesimal mathematics", The American mathematical monthly, vol. 104, no. 7, pp. 654-663, 1997, doi: 10.2307/2975060

[29] M. Mora, F. Córdova-Lepe, and R. Del-Valle, "A non-newtonian gradient for contour detection in images with multiplicative noise", Pattern recognition letters, vol. 33, no. 10, pp. 1245-1256, Jul. 2012, doi: 10.1016/j.patrec.2012.02.012

[30] S. Pawar, "The role of body size variation in community assembly", Advances in ecological research, vol. 52, pp. 201-248, 2015, doi: 10.1016/ bs.aecr.2015.02.003

[31] M. Peleg, M. G. Corradini, and M. D. Normand, "The logistic (Verhulst) model for sigmoid microbial growth curves revisited", Food research international, vol. 40, no. 7, pp. 808-818, Aug. 2007, doi: 10.1016/j.foodres.2007.01.012 
[32] O. Perron, "Die stabilitätsfrage bei differentialgleichungen", Mathematische zeitschrift, vol. 32, pp. 703-728, Dec. 1930, doi: 10.1007/ BF01194662

[33] M. Pinto and W. Campillay-Llanos, "Proportional differential equations", in VIII Congreso de Análisis Funcional y Ecuaciones de Evolución, Universidad de Santiago de Chile, 20 al 23 de Noviembre de 2013, Santiago, Chile. [On line]. Available: https:/ / bit.ly/ 3ea2jMw

[34] M. Pinto and W. Campillay-Llanos, "Proportional logistic growth", in 2do Workshop Modelamiento Matemático de Sistemas Biológicos, Universidad Tecnológica Metropolitana, 10 al 12 de Enero de 2018, Santiago Chile. [On line], Available: https:/ / bit.ly/ 3e8DctG

[35] V. D. Put and M. F Singer, Galois theory of linear differential equations, Berlin: Springer, 2003, doi: 10.1007/ 978-3-642-55750-7

[36] Slavik Antonín, Product integration, its history and applications, Prague: Matfyzpress, 2007. [On line]. Available: https:/ / bit.ly/ 35HZJcM

[37] V. Volterra and B. Hostinsky, Opérations infinitésimales linéaire, Paris: Gauthier-Villars, 1938.

[38] J. S. Weitz and S. A. Levin, "Size and scaling of predator-preydynamics", Ecology letters, vol. 9, no. 5, pp. 548-557, May 2006, doi: 10.1111/j.1461-0248.2006.00900.x

[39] C. P. Winsor, "The Gompertz curve as a growth curve", Proceedings of the national academy of sciences, vol. 18, no. 1, pp. 1-8, Jan. 1932, doi: 10.1073/ pnas.18.1.1

[40] T. Witelski and M. Bowen, Methods of mathematical modelling, Cham: Springer 2015. 